\title{
COMPUTATIONS IN FORMAL SYMPLECTIC GEOMETRY AND CHARACTERISTIC CLASSES OF MODULI SPACES
}

\author{
SHIGEYUKI MORITA, TAKUYA SAKASAI, AND MASAAKI SUZUKI
}

\begin{abstract}
We make explicit computations in the formal symplectic geometry of Kontsevich and determine the Euler characteristics of the three cases, namely commutative, Lie and associative ones, up to certain weights. From these, we obtain some nontriviality results in each case. In particular, we determine the integral Euler characteristics of the outer automorphism groups Out $F_{n}$ of free groups for all $n \leq 10$ and prove the existence of plenty of rational cohomology classes of odd degrees. We also clarify the relationship of the commutative graph homology with finite type invariants of homology 3-spheres as well as the leaf cohomology classes for transversely symplectic foliations. Furthermore we prove the existence of several new non-trivalent graph homology classes of odd degrees. Based on these computations, we propose a few conjectures and problems on the graph homology and the characteristic classes of the moduli spaces of graphs as well as curves.
\end{abstract}

\section{INTRODUCTION AND STATEMENTS OF THE MAIN RESULTS}

In celebrated papers [38][39], Kontsevich considered three infinite dimensional Lie algebras, namely commutative, Lie and associative ones. He proved that the stable homology group of each of these Lie algebras is isomorphic to a free graded commutative algebra generated by the stable homology group of $\mathfrak{s p}(2 g, \mathbb{Q})$ as $g$ tends to infinity together with certain set of generators which he described explicitly. It is the totality of the graph homologies for the commutative case, the totality of the cohomology groups of the outer automorphism groups of free groups, denoted by Out $F_{n}$, for the Lie case, and the totality of the cohomology groups of the moduli spaces of curves with unlabeled marked points, denoted by $\mathbf{M}_{g}^{m} / \mathfrak{S}_{m}$, for the associative case.

As for the commutative (resp. associative) case, Kontsevich described a general method of constructing cycles of the corresponding graph complex by making use of finite dimensional Lie (resp. $A_{\infty}$ ) algebras with non-degenerate invariant scalar products. In the Lie case, however, he mentioned that no non-trivial class was obtained by similar construction. In the associative case, he also introduced a dual construction of producing cocycles starting from a differential associative algebra with non-degenerate odd scalar product and trivial cohomology. Certain detailed description and generalizations of these methods have been given by several authors including Hamilton, Lazarev (see e.g. [33][32]) and others.

2000 Mathematics Subject Classification. Primary 17B40;17B56, Secondary 20J06; 55R40.

Key words and phrases. graph homology, symplectic derivation, automorphism groups of free groups, finite type invariants, transversely symplectic foliation, moduli space of curves. 
However there have been known only a few results which deduce new information about the graph homology and cohomology groups of Out $F_{n}$ or $\mathbf{M}_{g}^{m} / \mathfrak{S}_{m}$ by making a direct use of the above theorem of Kontsevich. First, as for the Lie case, in [50] the first named author defined a series of certain unstable homology classes of Out $F_{n}$ by using his trace maps introduced in [48]. Only the first three classes are known to be non-trivial (see Conant and Vogtmann [12] and Gray [31]). Second, recently Conant, Kassabov and Vogtmann [11] made a remarkable new development in this direction and defined many more classes. Thirdly, in the commutative case, the existence of two graph homology classes of odd degrees was proved, one in Gerlits [25] and the other in Conant, Gerlits and Vogtmann[10]. Fourthly, as for the associative case, in [52] a series of certain unstable homology classes for genus 1 moduli spaces was introduced, all of which have been proved to be non-trivial by Conant [9]. Finally, in our recent paper [54] we determined the stable abelianization of the Lie algebra in the associative case. As an application of this result, we obtained a new proof of an unpublished result of Harer. Church, Farb and Putman [7] gave a different proof.

The purpose of this paper is to continue these lines of investigations. We obtain new results in each of the three cases.

To be more precise, let $\Sigma_{g, 1}$ be a compact oriented surface of genus $g \geq 1$ with one boundary component and we denote its first rational homology group $H_{1}\left(\Sigma_{g, 1} ; \mathbb{Q}\right)$ simply by $H_{\mathbb{Q}}$. It can be regarded as the standard symplectic vector space of dimension $2 g$ induced from the intersection pairing on it. Let $\mathfrak{c}_{g}$ denote the graded Lie algebra consisting of Hamiltonian polynomial vector fields, without constant terms, on $H_{\mathbb{Q}} \otimes \mathbb{R} \cong \mathbb{R}^{2 g}$ with rational coefficients. The homogeneous degree $k$ part, denoted by $\mathfrak{c}_{g}(k)$, can be naturally identified with $S^{k+2} H_{\mathbb{Q}}$ where $S^{k} H_{\mathbb{Q}}$ denotes the $k$-th symmetric power of $H_{\mathbb{Q}}$. Let $\mathfrak{c}_{g}^{+}$be the ideal of $\mathfrak{c}_{g}$ consisting of Hamiltonian polynomial vector fields without linear terms. Next, we denote by $\mathcal{L}_{H_{\mathbb{Q}}}$ the free Lie algebra generated by $H_{\mathbb{Q}}$. Let $\mathfrak{h}_{g, 1}$ be the graded Lie algebra consisting of symplectic derivations of $\mathcal{L}_{H_{\mathbb{Q}}}$ and let $\mathfrak{h}_{g, 1}^{+}$be the ideal consisting of derivations with positive degrees. This Lie algebra was introduced in the theory of Johnson homomorphisms before the work of Kontsevich (see [47]) and has been investigated extensively. We use our notation for this Lie algebra. The notation $\mathfrak{h}_{g}$ is reserved for the case of a closed surface (see Remark 9.1) while $\mathfrak{h}_{g, 1}$ corresponds to genus $g$ compact surface with one boundary component.

Finally, let $T_{0} H_{\mathbb{Q}}$ denote the free associative algebra without unit generated by $H_{\mathbb{Q}}$. Let $\mathfrak{a}_{g}$ be the graded Lie algebra consisting of symplectic derivations of $T_{0} H_{\mathbb{Q}}$ and let $\mathfrak{a}_{g}^{+}$ be the ideal consisting of derivations with positive degrees.

We denote by $\operatorname{Sp}(2 g, \mathbb{Q})$ the symplectic group which we sometimes denote simply by Sp. If we fix a symplectic basis of $H_{\mathbb{Q}}$, then the space $H_{\mathbb{Q}}$ can be regarded as the standard representation of $\operatorname{Sp}(2 g, \mathbb{Q})$. Each piece $\mathfrak{c}_{g}(k), \mathfrak{h}_{g, 1}(k), \mathfrak{a}_{g}(k)$, of the three graded Lie algebras, is naturally an Sp-module so that it has an irreducible decomposition. It is known that this decomposition stabilizes when $g$ is sufficiently large.

Now we describe our main results. We determine the dimensions of the chain complexes which compute the Sp-invariant stable homology of the three Lie algebras 
$\mathfrak{c}_{g}^{+}, \mathfrak{h}_{g, 1}^{+}, \mathfrak{a}_{g}^{+}$up to certain weights (see Tables 1, 3 and 6). From this, we determine the Euler characteristic of each weight summand and the result is given as follows. For the definition of weight, see Section 2.

Theorem 1.1. The Euler characteristics $\chi$ of the Sp-invariant stable homologies of the three Lie algebras $\mathfrak{c}_{g}^{+}, \mathfrak{h}_{g, 1}^{+}, \mathfrak{a}_{g}^{+}$up to weight 20,18 or 16 are given as follows.

$$
\begin{gathered}
\text { (i) } \chi\left(H_{*}\left(\mathfrak{c}_{\infty}^{+}\right)_{w}^{\mathrm{Sp}}\right)=1,2,3,6,8,14,20,32,44,68 \quad(w=2,4, \ldots, 20) \\
\text { (ii) } \chi\left(H_{*}\left(\mathfrak{h}_{\infty}^{+}\right)_{w}^{\mathrm{Sp}}\right)=1,2,4,6,10,16,23,13,-96 \quad(w=2,4, \ldots, 18) \\
\text { (iii) } \chi\left(H_{*}\left(\mathfrak{a}_{\infty}^{+}\right)_{w}^{\mathrm{Sp}}\right)=2,5,12,24,50,100,188,347 \quad(w=2,4, \ldots, 16) .
\end{gathered}
$$

By combining Theorem 1.1 above with the description of the generators of the stable homologies due to Kontsevich, we obtain the following result. Part (ii) proves, in particular, the existence of odd dimensional rational homology classes of the outer automorphism groups of free groups for the first time.

Theorem 1.2. The integral Euler characteristics e of the primitive part of Sp-invariant stable homologies of the three Lie algebras $\mathfrak{c}_{g}^{+}, \mathfrak{h}_{g, 1}^{+}, \mathfrak{a}_{g}^{+}$, up to weight 20,18 or 16 , are given as follows.

$$
\begin{aligned}
& \text { (i) } e\left(G_{*}^{(n)}\right)=1,1,1,2,1,2,2,2,1,3 \quad(n=2,3, \ldots, 11 ; w=2 n-2) \\
& \text { (ii) } e\left(\text { Out } F_{n}\right)=1,1,2,1,2,1,1,-21,-124 \quad(n=2,3, \ldots, 10 ; w=2 n-2) \\
& \text { (iii) } \sum_{\substack{2 g-2+m=n \\
m>0}} e\left(\mathbf{M}_{g}^{m} / \mathfrak{S}_{m}\right)=2,2,4,2,6,6,6,1 \quad(n=1,2, \ldots, 8 ; w=2 n)
\end{aligned}
$$

Here $G_{*}^{(n)}$ denotes the graph complex due to Kontsevich which is defined in terms of graphs with the Euler characteristic $(1-n)$, Out $F_{n}$ denotes the outer automorphism group of the free group $F_{n}$ of rank $n, \mathbf{M}_{g}^{m}$ denotes the moduli space of curves of genus $g$ with labeled $m$ marked points and $\mathfrak{S}_{m}$ denotes the $m$-th symmetric group.

The commutative case of the graph homology has deep connections with two important subjects in topology. One is the theory of finite type invariants for homology 3-spheres initiated by Ohtsuki [59]. The other is the theory of characteristic classes of transversely symplectic foliations going back to Gelfand, Kalinin and Fuks [24] and more recently developed by Kontsevich [40] and further in [45][41]. On the other hand, a beautiful connection between these two theories was found by Garoufalidis and Nakamura [22].

Let $\mathcal{A}(\emptyset)$ denote the commutative algebra generated by vertex oriented connected trivalent graphs modulo the two relations, one is the (AS) relation and the other is the (IHX) relation. This algebra plays a fundamental role in the former theory above. In fact, Le [42] and Garoufalidis and Ohtsuki [23] proved that the graded algebra associated with the Ohtsuki filtration on the space of all the homology 3-spheres is isomorphic to $\mathcal{A}(\emptyset)$ which is a polynomial algebra generated by the subspaces $\mathcal{A}_{\text {conn }}^{(2 n-2)}(n=$ $2,3, \ldots)$ corresponding to connected graphs with $(2 n-2)$ vertices. Furthermore the 
completion $\widehat{\mathcal{A}}(\emptyset)$ of $\mathcal{A}(\emptyset)$ with respect to its gradings serves as the target of the LMO invariant introduced in [43].

As is well-known, the top homology group $H_{2 n-2}\left(G_{*}^{(n)}\right)$ of $G_{*}^{(n)}$ is canonically isomorphic to $\mathcal{A}_{\mathrm{conn}}^{(2 n-2)}$ because it can be seen that the top coboundary operator in the dual of the graph complex corresponds to the (IHX) relation (see Proposition 5.3). We can deduce from this fact that $\mathcal{A}(\emptyset)$ can be embedded into $H_{*}\left(\mathfrak{c}_{\infty}^{+}\right)^{\mathrm{Sp}}$ as a bigraded subalgebra. We define $\mathcal{E}$ to be the complementary bigraded algebra (see Definition 5.4 for details) so that we have an isomorphism $H_{*}\left(\mathfrak{c}_{\infty}^{+}\right)^{\mathrm{Sp}} \cong \mathcal{A}(\emptyset) \otimes \mathcal{E}$ of bigraded algebras. This bigraded algebra $\mathcal{E}$ can be interpreted as the space of all the graph homology classes represented by non-trivalent graphs. In the context of the theory of stable leaf cohomology classes for transversely symplectic foliations, it can also be interpreted as the dual space of all the exotic characteristic classes. Here by exotic we mean that the class depends on higher jets than the connection as well as the curvature forms by which the usual secondary characteristic classes are defined. See Section 5 for details.

Now we can deduce the following result from Theorem 1.2 (i).

Theorem 1.3. There exists an isomorphism

$$
H_{*}\left(\mathfrak{c}_{\infty}^{+}\right)^{\mathrm{Sp}} \cong \mathcal{A}(\emptyset) \otimes \mathcal{E}
$$

of bigraded algebras. If we denote by $P \mathcal{E}$ the primitive part of $\mathcal{E}$, then the Euler characteristic of its weight w-part $P \mathcal{E}_{w}$ is given by

$$
e\left(P \mathcal{E}_{w}\right)=0,0,0,0,-1,-1,-2,-3,-5,-5 \quad(w=2,4, \ldots, 20) .
$$

It follows that there exist several odd dimensional non-trivalent graph homology classes, as well as exotic stable leaf cohomology classes for transversely symplectic foliations, in each of the weights $w=10,12, \ldots, 20$.

The above theorem in the range $w \leq 10$ is essentially due to Gerlits [25], Theorem 4.1, and the case $w=12$ is due to Conant, Gerlits and Vogtmann [10], Theorem 5.1. In fact, in the former paper the author computed, among other things, $H_{*}\left(G_{*}^{(n)}\right)$ for all $n \leq 6$ and the case $n=7$ was treated in the latter paper. If we combine the former result with the above connection with the theory of foliations, we can conclude the existence of a certain exotic stable leaf cohomology class of transversely symplectic foliations of degree 7 and weight 10. This is the first appearance of such classes.

Next we consider Theorem 1.2 (ii). As is well-known, there is a beautiful formula for the rational Euler characteristics of the mapping class groups due to Harer-Zagier [34] and Penner [62]. In the case of Out $F_{n}$, Smillie and Vogtmann [65] obtained a generating function for the rational Euler characteristics $\chi\left(\right.$ Out $\left.F_{n}\right)$ and computed them for all $n \leq 100$. They are all negative and they conjecture that they are always negative. However, compared to the case of the mapping class groups, there still remain many open problems. For example, the relation between the rational and the integral Euler characteristics seems to be not very well understood. We will compare our computation above with the result of Smillie and Vogtmann cited above in Table 5 and we 
observe a very interesting behavior of the two numbers for the first time. See Section 6 for details of this as well as other discussions of our results.

Finally we consider the case of $\mathfrak{a}_{g}$. In this case, Kontsevich proved that the primitive part of $H_{*}\left(\mathfrak{a}_{\infty}^{+}\right)^{\mathrm{Sp}}$ corresponds to the totality of the $\mathfrak{S}_{m}$-invariant rational cohomology groups $H^{*}\left(\mathbf{M}_{g}^{m} ; \mathbb{Q}\right)^{\mathfrak{S}_{m}}$ of the moduli spaces $\mathbf{M}_{g}^{m}$ of genus $g$ curves with $m$ marked points for all $g, m$ with $2 g-2+m>0, m \geq 1$. There have been known many results concerning the cohomology of these moduli spaces for the cases of low genera $g=0,1,2,3,4$ due to Getzler-Kapranov [28], Getzler [26][27], Looijenga [44], Tommasi [67][68], Gorsky [29][30], Bergström [1] and others. In Section 7, we will check that our computation of the Euler characteristics is consistent with these known results or deduced from them by explicit computations, in the range $2 g-2+m \leq 8$.

In our forthcoming paper [56], which is a sequel to this paper, we will extend both of Theorem 1.1 (iii) and Theorem 1.2 (iii) from $w=16$ to 500 by adopting a completely different method. More precisely, we use a formula of Gorsky [30] for the equivariant Euler characteristics of the moduli space of curves to obtain certain closed formulas which enable us to determine the above values. However, in this paper we only describe the values which we deduced from Table 6 in order to compare with the other two cases. See Section 7 for more details about this point.

To compute the graph homologies directly, we have to enumerate certain types of graphs. However, according to the number of vertices increases, the difficulty of the problem of deciding whether two given graphs are isomorphic to each other grows very rapidly. In view of this, we adopted a method in a pure framework of symplectic representation theory. This has a disadvantage that the dimensions which we have to compute are considerably larger than the graph theoretical method, because there is no effective way to distinguish between connected and disconnected graphs in the framework of representation theory. In order to overcome this difficulty, we made various devices to lighten the burden imposed on computers. More precisely, our task is to determine the dimensions of the Sp-invariant subspaces of various Sp-modules. Theoretically there is no problem here because we know the characters of these modules completely. However, the problem lies in the huge size of the data as well as the time which computers need. We have developed several our own programs on the computer software Mathematica which realize theoretical considerations in the representation theory. See Section 4 for details. We have also made an extensive use of the computer program LiE to obtain irreducible decompositions of various Sp-modules.

Acknowledgement. The authors would like to thank Christophe Soule for helpful information about the (non)vanishing of the Borel regulator classes. We also thank James Conant and Alastair Hamilton for informing the authors about their works [10] and [32]. Thanks are also due to a referee for pointing out that Proposition 5.3 is wellknown. The first named author would like to thank Richard Hain and Hiroaki Nakamura for enlightening discussions about the symplectic representation theory related to the mapping class group in the 1990's. The authors were partially supported by 
KAKENHI (No. 24740040 and No. 24740035), Japan Society for the Promotion of Science, Japan.

\section{PRELIMINARIES}

In this section, we prepare a few facts about the (co)homology of graded Lie algebras which will be needed in our later considerations.

Let $\mathfrak{g}=\oplus_{k=0}^{\infty} \mathfrak{g}(k)$ be a graded Lie algebra over $\mathbb{Q}$ and let $\mathfrak{g}^{+}=\oplus_{k=1}^{\infty} \mathfrak{g}(k)$ be its ideal consisting of all the elements of $\mathfrak{g}$ with positive gradings. We assume that each piece $\mathfrak{g}(k)$ is finite dimensional for all $k$. Then the chain complex $C_{*}(\mathfrak{g})$ of $\mathfrak{g}$ splits into the direct sum

$$
C_{*}(\mathfrak{g})=\bigoplus_{w=0}^{\infty} C_{*}^{(w)}(\mathfrak{g})
$$

of finite dimensional subcomplexes $C_{*}^{(w)}(\mathfrak{g})=\bigoplus_{i=0}^{w} C_{i}^{(w)}(\mathfrak{g})$ where

$$
C_{i}^{(w)}(\mathfrak{g})=\bigoplus_{\substack{i_{0}+i_{1}+\cdots+i_{w}=i \\ i_{1}+2 i_{2}+\cdots+w i_{w}=w}} \wedge^{i_{0}}(\mathfrak{g}(0)) \otimes \wedge^{i_{1}}(\mathfrak{g}(1)) \otimes \cdots \otimes \wedge^{i_{w}}(\mathfrak{g}(w))
$$

so that $C_{i}^{(w)}(\mathfrak{g})=0$ for $i>w+\frac{1}{2} d(d-1)(d=\operatorname{dim} \mathfrak{g}(0))$. This induces a bigraded structure on the homology group $H_{*}(\mathfrak{g})$ described as

$$
H_{i}(\mathfrak{g})=\bigoplus_{w=0}^{\infty} H_{i}(\mathfrak{g})_{w}
$$

where $H_{i}(\mathfrak{g})_{w}=H_{i}\left(C_{*}^{(w)}(\mathfrak{g})\right)$. We call $H_{i}(\mathfrak{g})_{w}$ the weight $w$-part of $H_{i}(\mathfrak{g})$. Let $\widehat{\mathfrak{g}}$ be the completion of $\mathfrak{g}$ with respect to the grading and let $H_{c}^{*}(\widehat{\mathfrak{g}})$ be the continuous cohomology. Then we have

$$
H_{c}^{k}(\widehat{\mathfrak{g}}) \cong \bigoplus_{w=0}^{\infty}\left(H_{k}(\mathfrak{g})_{w}\right)^{*}
$$

Now suppose that $\mathfrak{g}$ is an Sp-graded Lie algebra by which we mean that each piece $\mathfrak{g}(k)$ is a finite dimensional representation of $\operatorname{Sp}(2 g, \mathbb{Q})$ for some fixed $g$ such that the bracket operation $\mathfrak{g}(i) \otimes \mathfrak{g}(j) \rightarrow \mathfrak{g}(i+j)$ is a morphism of Sp-modules for any $i, j$. We further assume that $\mathfrak{g}(0)=\mathfrak{s p}(2 g, \mathbb{Q}) \cong S^{2} H_{\mathbb{Q}}$. Then we have a split extension

$$
0 \rightarrow \mathfrak{g}^{+} \rightarrow \mathfrak{g} \rightarrow \mathfrak{s p}(2 g, \mathbb{Q}) \rightarrow 0
$$

of Lie algebras. The $E^{2}$-term of the Hochschild-Serre spectral sequence for the homology of $\mathfrak{g}$ is given by

$$
E_{p, q}^{2}=H_{p}\left(\mathfrak{s p}(2 g, \mathbb{Q}) ; H_{q}\left(\mathfrak{g}^{+}\right)\right) .
$$

By the assumption, the chain complex $C_{*}\left(\mathfrak{g}^{+}\right)$decomposes into the direct sum of subcomplexes corresponding to Sp-irreducible components. It follows that the homology group $H_{q}\left(\mathfrak{g}^{+}\right)$also decomposes into the Sp-irreducible components. In particular, we have the Sp-invariant part which we denote by $H_{q}\left(\mathfrak{g}^{+}\right)^{\text {Sp }}$. Here we can apply the well-known vanishing theorem (see Chevalley and Eilenberg [6]) to conclude that 
$H_{p}\left(\mathfrak{s p}(2 g, \mathbb{Q}) ; H_{q}\left(\mathfrak{g}^{+}\right)_{\lambda}\right)=0$ for any $p \geq 0$ and for any Sp-irreducible component $\lambda$ different from the trivial representation. It follows that the spectral sequence collapses at the $E^{2}$-term and we have an isomorphism

$$
H_{*}(\mathfrak{g}) \cong H_{*}(\mathfrak{s p}(2 g ; \mathbb{Q})) \otimes H_{*}\left(\mathfrak{g}^{+}\right)^{\mathrm{Sp}} .
$$

This argument can be applied in the case of three Lie algebras treated in this paper. More precisely, we set $\mathfrak{g}_{g}$ to be one of $\mathfrak{c}_{g}, \mathfrak{h}_{g, 1}$ or $\mathfrak{a}_{g}$. Then we have natural embeddings $\mathfrak{g}_{g} \subset \mathfrak{g}_{g+1}$ so that we can consider the union (or equivalently the direct limit) $\mathfrak{g}_{\infty}=$ $\lim _{g \rightarrow \infty} \mathfrak{g}_{g}$. Also we have its ideal $\mathfrak{g}_{\infty}^{+}$. The homology groups of them are given by

$$
H_{*}\left(\mathfrak{g}_{\infty}\right)=\lim _{g \rightarrow \infty} H_{*}\left(\mathfrak{g}_{g}\right), \quad H_{*}\left(\mathfrak{g}_{\infty}^{+}\right)=\lim _{g \rightarrow \infty} H_{*}\left(\mathfrak{g}_{g}^{+}\right) .
$$

Since it is well known that the Sp-irreducible decompositions of $\mathfrak{g}_{g}(k)$ stabilizes as $g$ goes to $\infty$, we can apply the preceding argument to conclude that

$$
H_{*}\left(\mathfrak{g}_{\infty}\right) \cong H_{*}(\mathfrak{s p}(\infty, \mathbb{Q})) \otimes H_{*}\left(\mathfrak{g}_{\infty}^{+}\right)^{\mathrm{Sp}}, \quad H_{*}\left(\mathfrak{g}_{\infty}^{+}\right)^{\mathrm{Sp}}=\bigoplus_{w=1}^{\infty} H_{*}\left(\mathfrak{g}_{\infty}^{+}\right)_{w}^{\mathrm{Sp}}
$$

Similar formulas are also valid for the continuous cohomology, although we have to be careful here because the projective limit arises rather than the direct limit.

Since $H_{*}\left(\mathfrak{g}_{\infty}^{+}\right)_{w}^{\mathrm{Sp}}$ is finite dimensional by the assumption, we have its Euler characteristic $\chi\left(H_{*}\left(\mathfrak{g}_{\infty}^{+}\right)_{w}^{\mathrm{Sp}}\right)$. We call

$$
g(t)=1+\sum_{w=1}^{\infty} \chi\left(H_{*}\left(\mathfrak{g}_{\infty}^{+}\right)_{w}^{\mathrm{Sp}}\right) t^{w} \in \mathbb{Z}[[t]]
$$

the weight generating function for the Sp-invariant stable homology group of the Sp-Lie algebra $\mathfrak{g}^{+}$. Observe here that if we replace $\mathfrak{g}^{+}$with $\mathfrak{g}$ here, then we obtain the trivial function 1 because $\chi\left(H_{*}(\mathfrak{s p}(2 g, \mathbb{Q}))\right)=0$.

Remark 2.1. We mention that such a kind of generating function was first considered by Perchik [62] in the context of the unstable Gelfand-Fuks cohomology of the Lie algebra of formal Hamiltonian vector fields $\left(\mathfrak{h a m} \mathfrak{m}_{2 g}\right.$ in the notation of [40] and Section 5 below) and later in [41] in the context of the stable Gelfand-Fuks cohomology of $\lim _{g \rightarrow \infty} \mathfrak{h a m}_{2 g}^{0}=\widehat{\mathfrak{c}}_{\infty} \otimes \mathbb{R}$.

In the case where $\mathfrak{g}_{g}$ is one of the three Lie algebras considered in this paper, we can consider the weight generating function for the Sp-invariant homology group of the limit Lie algebra $\mathfrak{g}_{\infty}^{+}$.

For later use, we generalize the definition of the weight generating function in a broader context as follows.

Let

$$
\mathcal{K}=\bigoplus_{d, w=0}^{\infty} \mathcal{K}_{d, w}
$$

be a bigraded algebra over $\mathbb{Q}$ such that the multiplication

$$
\mathcal{K}_{d, w} \otimes \mathcal{K}_{d^{\prime}, w^{\prime}} \longrightarrow \mathcal{K}_{d+d^{\prime}, w+w^{\prime}}
$$


is graded commutative with respect to $d$ (called the degree) and the weight $w$ part

$$
\mathcal{K}_{w}=\bigoplus_{d} \mathcal{K}_{d, w}
$$

is finite dimensional for any $w$. We define the Euler characteristic $\chi\left(\mathcal{K}_{w}\right)$ by

$$
\chi\left(\mathcal{K}_{w}\right)=\sum_{d}(-1)^{d} \operatorname{dim} \mathcal{K}_{d, w} .
$$

We also assume that $\mathcal{K}_{0,0}=\mathbb{Q}$. Hereafter we always assume the above conditions whenever we mention weight generating functions of bigraded algebras. It is easy to see that if there are given two bigraded algebras $\mathcal{K}, \mathcal{K}^{\prime}$ which satisfy the above conditions, then the tensor product $\mathcal{K} \otimes \mathcal{K}^{\prime}$ also satisfies them with respect to the induced bigradings on it.

Definition 2.2. We define the weight generating function $k(t)$ for a bigraded algebra $\mathcal{K}$ as above to be

$$
k(t)=1+\sum_{w=1}^{\infty} \chi\left(\mathcal{K}_{w}\right) t^{w} .
$$

Example 2.3. Let $P=\mathbb{Q}\left[x_{1}, x_{2}, \ldots\right]$ be the polynomial algebra on given variables $x_{i}$ with degree $2 d_{i}$. If we set the weight to be equal to the degree, then the weight generating function for $P$ is given by

$$
p(t)=\prod_{i}\left(1+t^{2 d_{i}}+t^{4 d_{i}}+\cdots\right)=\prod_{i}\left(1-t^{2 d_{i}}\right)^{-1} .
$$

Let $E=\wedge^{*}\left[y_{1}, y_{2}, \ldots\right]$ be the exterior algebra on given variables $y_{i}$ with degree $2 s_{i}-1$. If we set the weight to be equal to the degree, then the weight generating function for $E$ is given by

$$
e(t)=\prod_{i}\left(1-t^{2 s_{i}-1}\right)
$$

Proposition 2.4. Let $\mathcal{K}$ be a bigraded algebra and assume that it is a free graded commutative algebra with respect to the grading by degrees. Let $P \mathcal{K}$ be the subspace consisting of primitive elements. For each $d, w$, set $P \mathcal{K}_{d, w}=P \mathcal{K} \cap \mathcal{K}_{d, w}$ and define

$$
\chi_{w}^{\mathrm{pr}}(\mathcal{K})=\sum_{d}(-1)^{d} \operatorname{dim} P \mathcal{K}_{d, w} .
$$

Then we have

$$
k(t)=\prod_{w=1}^{\infty}\left(1-t^{w}\right)^{-\chi_{w}^{\mathrm{pr}}(\mathcal{K})} .
$$

Proof. It is easy to see that the weight generating function of the tensor product of two graded commutative bialgebras is the product of those of each bigraded algebra. Since $\mathcal{K}$ is free by the assumption, it is the tensor product of subalgebras generated by $P \mathcal{K}$. Consider the product of weight generating functions of two graded commutative algebras each of which is generated by an element whose weight is the same whereas the degree is complementary, namely one is even and the other is odd. Then by Example 
2.3, we see that this product is the constant function 1 . Hence the weight generating function of $\mathcal{K}$ depends only on $\chi_{w}^{\mathrm{pr}}(\mathcal{K})$ and the claim follows.

Remark 2.5. The number $\chi_{w}^{\mathrm{pr}}(\mathcal{K})$ can be interpreted as the Euler characteristic of the space of primitive elements of weight $w$, or equivalently as the number of new generators of weight $w$ with even degrees minus that of new generators of weight $w$ with odd degrees.

Proposition 2.6. Let $\mathcal{K}$ be a bigraded algebra and assume that it is a free graded commutative algebra with respect to the grading by degrees. Assume that the weight generating function $k(t)$ is determined up to weight $w_{0}$. Then we can determine the numbers $\chi_{w}^{\mathrm{pr}}(\mathcal{K})\left(w=1,2, \ldots, w_{0}\right)$ inductively by the following recursive formula

$$
\chi_{w}^{\mathrm{pr}}(\mathcal{K})=[k(t)]_{t^{w}}-\left[\prod_{i=1}^{w-1}\left(1-t^{i}\right)^{-\chi_{i}^{\mathrm{pr}}(\mathcal{K})}\right]_{t^{w}} \quad\left(w=1,2, \ldots, w_{0}\right)
$$

where $[f(t)]_{t^{w}}$ denotes the coefficient of $t^{w}$ in a given formal power series $f(t) \in \mathbb{Z}[[t]]$.

Proof. This follows from Proposition 2.4

\section{COMPUtATION OF THE IRREDUCIBLE DECOMPOSITIONS}

In this section we describe our explicit determination of the stable irreducible decompositions of $\mathfrak{h}_{g, 1}(k), \mathfrak{a}_{g}(k)$ up to certain degrees. At present, we have determined them for all $k \leq 20$. As already mentioned in the introduction, in the commutative case, we have an isomorphism $\mathfrak{c}_{g}(k) \cong S^{k+2} H_{\mathbb{Q}}$ which is known to be an irreducible representation for any $k$.

To describe our result, we fix our notations. Any Young diagram $\lambda=\left[\lambda_{1} \cdots \lambda_{h}\right]$ with $k$ boxes defines an irreducible representation of the symmetric group $\mathfrak{S}_{k}$ which we denote by $\lambda_{\mathfrak{S}_{k}}$ or sometimes simply by the same symbol $\lambda$. Thus $[k]$ corresponds to the trivial representation and $\left[1^{k}\right]$ the alternating representation. Here we use a simplified notation to express Young diagrams. For example [422] will be denoted by $\left[42^{2}\right]$. For any Young diagram $\lambda=\left[\lambda_{1} \cdots \lambda_{h}\right]$ as above and for any $n \geq h$, let $\lambda_{\mathrm{GL}}$ be the corresponding irreducible representation of $\operatorname{GL}(n, \mathbb{Q})$. Similarly for any $g \geq h$, let $\lambda_{\mathrm{Sp}}$ be the corresponding irreducible representation of $\operatorname{Sp}(2 g, \mathbb{Q})$.

Our method of computing the stable irreducible decompositions for the Lie case $\mathfrak{h}_{g, 1}$ as well as the associative case $\mathfrak{a}_{g}$ can be described as follows. For the former case, we use the following result of Kontsevich.

Theorem 3.1 (Kontsevich [38][39]). Let $W_{k}$ be the $\mathfrak{S}_{k+2}$-module with character $\chi_{k}\left(1^{k+2}\right)=$ $k !, \chi_{k}\left(1^{1} a^{b}\right)=(b-1) ! a^{b-1} \mu(a), \chi_{k}\left(a^{b}\right)=-(b-1) ! a^{b-1} \mu(a)$, and $\chi_{k}$ vanishes on all the other conjugacy classes, where $\mu$ denotes the Möbius function. Then there exists an isomorphism

$$
\mathfrak{h}_{g, 1}(k) \cong H_{\mathbb{Q}}^{\otimes(k+2)} \otimes_{A_{k+2}} W_{k}
$$

of $\operatorname{Sp}(2 g, \mathbb{Q})$-modules, where $A_{k+2}=\mathbb{Q} \mathfrak{S}_{k+2}$ denotes the group algebra of $\mathfrak{S}_{k+2}$. 
We have given in [55] a simple proof of this result using only the standard representation theory (see also [16]). As an immediate corollary to this theorem, we obtain the following.

Corollary 3.2. Let $\lambda=\left[\lambda_{1} \cdots \lambda_{h}\right]$ be a Young diagram with $(k+2)$ boxes and let $\lambda_{\mathfrak{S}_{k+2}}$ be the corresponding irreducible representation of the symmetric group $\mathfrak{S}_{k+2}$. Then the multiplicity $m_{\lambda}$ of $V_{\lambda}=H_{\mathbb{Q}}^{\otimes(k+2)} \otimes_{A_{k+2}} \lambda_{\mathfrak{S}_{k+2}}$ in $\mathfrak{h}_{g, 1}(k)$ is expressed as

$$
m_{\lambda}=\frac{1}{(k+2) !} \sum_{\gamma \in \mathfrak{S}_{k+2}} \chi_{k}(\gamma) \chi_{\lambda}(\gamma)
$$

where $\chi_{\lambda}$ denotes the character of $\lambda_{\mathfrak{S}_{k+2}}$.

For the case of $\mathfrak{a}_{g}(k)$, we have the following result.

Proposition 3.3. Let $\lambda=\left[\lambda_{1} \cdots \lambda_{h}\right]$ be a Young diagram with $(k+2)$ boxes and let $\lambda_{\mathfrak{S}_{k+2}}$ be the corresponding irreducible representation of the symmetric group $\mathfrak{S}_{k+2}$. Then the multiplicity $n_{\lambda}$ of $V_{\lambda}=H_{\mathbb{Q}}^{\otimes(k+2)} \otimes_{A_{k+2}} \lambda_{\mathfrak{S}_{k+2}}$ in $\mathfrak{a}_{g}(k)$ is expressed as

$$
n_{\lambda}=\frac{1}{k+2} \sum_{i=1}^{k+2} \chi_{\lambda}\left(\sigma_{k+2}^{i}\right)
$$

where $\sigma_{k+2} \in \mathfrak{S}_{k+2}$ denotes the cyclic permutation $(12 \cdots(k+2))$ of order $k+2$.

Proof. As is well known, for any $k$ we have an isomorphism

$$
H_{\mathbb{Q}}^{\otimes(k+2)} \cong \bigoplus_{|\lambda|=k+2}\left(\operatorname{dim} \lambda_{\mathfrak{S}_{k+2}}\right) V_{\lambda}
$$

as $\mathrm{GL}(2 g, \mathbb{Q})$-modules, where $|\lambda|$ denotes the number of the boxes of the Young diagram $\lambda$. On the other hand, we have an isomorphism

$$
\mathfrak{a}_{g}(k)=\left(H_{\mathbb{Q}}^{\otimes(k+2)}\right)^{\mathbb{Z} /(k+2)}
$$

where the cyclic group $\mathbb{Z} /(k+2)$ of order $k+2$ acts on $H_{\mathbb{Q}}^{\otimes(k+2)}$ by cyclic permutations. Then the claim follows by considering the restriction to the subgroup $\mathbb{Z} /(k+2) \subset \mathfrak{S}_{k+2}$ and applying the standard argument.

Our explicit irreducible decompositions of $\mathfrak{h}_{g, 1}(k)$ and $\mathfrak{a}_{g}(k)$ as $\operatorname{Sp}(2 g, \mathbb{Q})$-modules are done as follows. First we determine the irreducible decompositions of these modules as $\mathrm{GL}(2 g, \mathbb{Q})$-modules. For this, we use Corollary 3.2 and Proposition 3.3, respectively, to compute the multiplicities $m_{\lambda}, n_{\lambda}$ by applying the formula of Frobenius which expresses the value $\chi_{\lambda}(\gamma)$ for any given element $\gamma \in \mathfrak{S}_{k+2}$ as the coefficient of a certain polynomial $f_{\lambda}$ with respect to a certain monomial $x_{\lambda}$ (see e.g. [19], Frobenius Formula 4.10). We made a systematic computer computation by using this formula. Recall here that we must take the number of variables for the polynomial $f_{\lambda}$ at least as large as the number $h(\lambda)$ of rows of the Young diagram $\lambda$. Hence, the necessary data will get larger and larger as the number $h(\lambda)$ increases. To overcome this difficulty, we adopted the 
following simple argument. Let $\lambda^{\prime}$ denote the conjugate Young diagram of any given one $\lambda$. Then, as is well known, we have an isomorphism

$$
\lambda_{\mathfrak{S}_{k+2}}^{\prime} \cong \lambda_{\mathfrak{S}_{k+2}} \otimes\left[1^{k+2}\right]
$$

where $\left[1^{k+2}\right]$ denotes the 1 -dimensional alternating representation. Since, for any given $\mathfrak{S}_{k+2}$-module $W$, its multiplicity of an irreducible representation $\lambda$ is equal to that of $\lambda^{\prime}$ in the conjugate representation $W^{\prime}=W \otimes\left[1^{k+2}\right]$, we can easily deduce the following.

Proposition 3.4. In the same situation as in Corollary 3.2 and Proposition 3.3, we have

$$
\begin{gathered}
m_{\lambda}=\frac{1}{(k+2) !} \sum_{\gamma \in \mathfrak{S}_{k+2}}(\operatorname{sgn} \gamma) \chi_{k}(\gamma) \chi_{\lambda^{\prime}}(\gamma) \\
n_{\lambda}=\frac{1}{k+2} \sum_{i=1}^{k+2}\left(\operatorname{sgn} \sigma_{k+2}^{i}\right) \chi_{\lambda^{\prime}}\left(\sigma_{k+2}^{i}\right)
\end{gathered}
$$

where $\operatorname{sgn} \gamma$ denotes the sign of $\gamma$.

Since the two numbers $h\left(\lambda^{\prime}\right)$ and $h(\lambda)$ are so to speak complementary to each other (e.g. $h\left(\left[1^{k}\right]^{\prime}\right)=1$ while $h\left(\left[1^{k}\right]\right)=k$ ), we can make computer computations roughly twice as much compared to the situation where we do not use this method. Also certain symmetries in the structure of $\mathfrak{h}_{g, 1}$ as well as $\mathfrak{a}_{g}$ which we found in [55] decrease necessary computations considerably.

Next we use the known formula of decomposing a given irreducible $\operatorname{GL}(2 g, \mathbb{Q})$ module $\lambda_{G L}$ into $\operatorname{Sp}(2 g, \mathbb{Q}$ )-irreducible components (see e.g. formula (25.39) in [19]). We made a computer program of this formula and by using it we made a database which contains the $\operatorname{Sp}(2 g, \mathbb{Q})$-irreducible decomposition of all the $\mathrm{GL}(2 g, \mathbb{Q})$-modules $\lambda_{G L}$ with the number $|\lambda|$ of boxes of the Young diagram $\lambda$ less than or equal to 30 (there are 28628 such Young diagrams).

As mentioned already, we have so far determined the $\operatorname{Sp}(2 g, \mathbb{Q})$-irreducible decompositions of $\mathfrak{h}_{g, 1}(k)$ and $\mathfrak{a}_{g}(k)$ for all $k \leq 20$ by making use of the above method. Although here we omit the description of the results, see Tables 8 and 9 in which we express the dimensions of the Sp-invariant subspaces $\mathfrak{h}_{g, 1}(k)^{\mathrm{Sp}}$ for all $k \leq 20$. These tables contain more precise information on these subspaces. Namely they contain a complete description how these subspaces degenerate according as the genus $g$ decreases from the stable range one by one to the final case $g=1$.

\section{COMPUTATION OF THE Dimensions OF THE Sp-INVARIANT SUbSPACES OF VARIOUS Sp-MODULES}

In this section, we describe several methods which we developed in our computer computations. We have to determine the dimensions of the subspaces consisting of the Sp-invariant elements of various Sp-modules such as

$$
\left(\wedge^{d_{1}} \mathfrak{c}_{g}\left(i_{1}\right)\right) \otimes \cdots \otimes\left(\wedge^{d_{s}} \mathfrak{c}_{g}\left(i_{s}\right)\right)
$$

or the corresponding modules where we replace $\mathfrak{c}_{g}$ by $\mathfrak{h}_{g, 1}$ or $\mathfrak{a}_{g}$. We mention that the character of any of these modules is known so that theoretically there is no problem. 
More precisely, we can adopt the method given in [41], which treated the case of $\mathfrak{c}_{g}$ by extending the original one due to Perchik [62], to the other two case as well to obtain closed formulas for the above dimensions of Sp-invariants. Unfortunately however, these formulas are too complicated so that when we use computers to obtain explicit values, the memory problem arises in a very early stage. We have developed other methods described as follows.

Method (I) (GL-decomposition of tensor products)

There is a formula, called the Littlewood-Richardson rule, which gives the irreducible decomposition of the tensor product of any two GL-modules and a similar formula is known for the case of Sp-modules (see e.g. [19]). However, the latter formula is considerably more complicated than the former one. In view of this, we postpone the Sp-irreducible decomposition as late as possible and we make the GL-irreducible decomposition as far as possible. We made a computer program for the LittlewoodRichardson rule and apply it in various stages in our computation.

\section{Method (II) (Sp-decomposition of GL-modules)}

There is a combinatorial formula which gives the Sp-irreducible decomposition of any irreducible GL-module $\lambda_{\mathrm{GL}}$, namely the restriction law corresponding to the pair $\operatorname{Sp}(2 g, \mathbb{Q}) \subset \mathrm{GL}(2 g, \mathbb{Q})$ (see [19]). We made a computer program for this procedure and apply it in various stages in our work.

Method (III) (Counting the number of Young diagrams with multiple double floors)

Let us call a Young diagram $\lambda$ with multiple double floors if it has the form $\lambda=$ $\left[\lambda_{1} \lambda_{1} \cdots \lambda_{s} \lambda_{s}\right]$. It is easy to see that $\lambda$ is such a Young diagram if and only if its conjugate Young diagram $\lambda^{\prime}$ is of even type in the sense that all the numbers appearing in it are even integers. Now at the final stage of counting the dimension of the Spinvariant subspace of a GL-module $V$, we can determine the required number without performing the Sp-decomposition of $V$ by adopting the following method.

Proposition 4.1. Let $V$ be a $\mathrm{GL}(2 g, \mathbb{Q})$-module and let $V^{\mathrm{Sp}}$ denote the subspace consisting of $\operatorname{Sp}(2 g, Q)$-invariant elements of $V$ considered as an $\operatorname{Sp}(2 g, \mathbb{Q})$-module. Also let

$$
V=\bigoplus_{\lambda} m_{\lambda}(V) \lambda_{\mathrm{Sp}}
$$

be the Sp-irreducible decomposition of $V$. Then we have the equality

$$
\operatorname{dim} V^{\mathrm{Sp}}=\sum_{\lambda: \text { multiple double floors }} m_{\lambda}(V) .
$$

Proof. This follows from the fact that

$$
\operatorname{dim}\left(\lambda_{\mathrm{GL}}\right)^{\mathrm{Sp}}= \begin{cases}1 & (\lambda: \text { multiple double floors }) \\ 0 & \text { (otherwise })\end{cases}
$$


which follows from the restriction law corresponding to the pair $\operatorname{Sp}(2 g, \mathbb{Q}) \subset \mathrm{GL}(2 g, \mathbb{Q})$.

We made a computer program which counts the number of Young diagrams with multiple double floors in any linear combination of Young diagrams.

Method (IV) (Counting pairs of Young diagrams with the same shape)

The difficulty in applying our program of performing the Littlewood-Richardson rule for the tensor product $V_{1} \otimes V_{2}$ increases according to the numbers of boxes of the Young diagrams appearing in the irreducible decompositions of $V_{i}$ get larger and larger. In case we cannot obtain the result within an appropriate time, we adopt this method which depends on the following fact.

Proposition 4.2. Let $V_{1}, V_{2}$ be two $\mathrm{GL}(2 g, \mathbb{Q})$-modules and let

$$
V_{i}=\bigoplus_{\lambda} m_{\lambda}\left(V_{i}\right) \lambda_{\mathrm{Sp}} \quad(i=1,2)
$$

be the Sp-irreducible decompositions of $V_{i}$. Then we have the equality

$$
\operatorname{dim}\left(V_{1} \otimes V_{2}\right)^{\mathrm{Sp}}=\sum_{\lambda} m_{\lambda}\left(V_{1}\right) m_{\lambda}\left(V_{2}\right) .
$$

Proof. This follows from the well known fact that

$$
\operatorname{dim}\left(\lambda_{\mathrm{Sp}} \otimes \mu_{\mathrm{Sp}}\right)^{\mathrm{Sp}}=\left\{\begin{array}{ll}
1 & (\lambda=\mu) \\
0 & \text { (otherwise) }
\end{array} .\right.
$$

Here is another similar formula.

Proposition 4.3. Let $V$ be an $\operatorname{Sp}(2 g, \mathbb{Q})$-module and let

$$
V=\bigoplus_{\lambda} m_{\lambda}(V) \lambda_{\mathrm{Sp}}
$$

be its Sp-irreducible decomposition. Then we have the equality

$$
\operatorname{dim}\left(\wedge^{2} V\right)^{\mathrm{Sp}}=\frac{1}{2}\left(\sum_{|\lambda|: \text { odd }} m_{\lambda}(V)\left(m_{\lambda}(V)+1\right)+\sum_{|\lambda|: \text { even }}\left(m_{\lambda}(V)-1\right) m_{\lambda}(V)\right) .
$$

Proof. This follows from the well known fact that

$$
\operatorname{dim}\left(\wedge^{2} \lambda_{\mathrm{Sp}}\right)^{\mathrm{Sp}}=\left\{\begin{array}{ll}
1 & (|\lambda|: \text { odd }) \\
0 & (|\lambda| \text { : even })
\end{array} .\right.
$$

We made a computer program which counts the number of pairs with the same Young diagrams in any two linear combination of Young diagrams. We can use this method to check the accuracy of our computations by applying it to plural expressions

$$
V=V_{1} \otimes V_{2}=V_{1}^{\prime} \otimes V_{2}^{\prime}
$$


as tensor products of the same $\mathrm{GL}(2 g, \mathbb{Q})$-module $V$.

Method (V) (Adams operations)

The most difficult part in our computation is the determination of the GL as well as Sp irreducible decomposition of the exterior powers $\wedge^{k}\left[1^{3}\right]_{\mathrm{GL}}$ of $\mathfrak{h}_{g, 1}(1)=\left[1^{3}\right]_{\mathrm{GL}}$.

As is well known, the character of $\wedge^{k}[1]_{\mathrm{GL}}=\left[1^{k}\right]_{\mathrm{GL}}$ is given by

$$
\operatorname{ch}\left(\left[1^{k}\right]_{\mathrm{GL}}\right)=E_{k}\left(x_{1}, \ldots, x_{N}\right)
$$

where $E_{k}$ denotes the $k$-th elementary symmetric polynomial and $N$ denotes some fixed large number. In particular $\operatorname{ch}\left(\left[1^{3}\right]_{\mathrm{GL}}\right)=E_{3}$. Then the character of $\wedge^{k}\left[1^{3}\right]_{\mathrm{GL}}$ can be written as

$$
\operatorname{ch}\left(\wedge^{k}\left[1^{3}\right]_{\mathrm{GL}}\right)=E_{k}\left(E_{3}\right)
$$

where $E_{k}\left(E_{3}\right)$ denotes the $k$-th elementary symmetric polynomial with respect to the new variables $\left\{x_{i} x_{j} x_{k}\right\}_{i<j<k}$. Here we apply the well-known classical combinatorial algorithm to express any symmetric polynomial as a polynomial on the elementary symmetric polynomial to obtain a formula for the character of $\wedge^{k}\left[1^{3}\right]_{\text {GL }}$. For example $\operatorname{ch}\left(\wedge^{2}\left[1^{3}\right]_{\mathrm{GL}}\right)=E_{6}+E_{2} E_{4}-E_{1} E_{5}$. Then we apply Method (I) to obtain the GLirreducible decomposition and further apply Method (II) to obtain the Sp-irreducible decomposition. For example we have

$$
\begin{aligned}
\wedge^{2}\left[1^{3}\right]_{\mathrm{GL}} & =\left[1^{6}\right]_{\mathrm{GL}}+\left[2^{2} 1^{2}\right]_{\mathrm{GL}} \\
& =\left[1^{6}\right]_{\mathrm{Sp}}+\left[2^{2} 1^{2}\right]_{\mathrm{Sp}}+2\left[1^{4}\right]_{\mathrm{Sp}}+\left[21^{2}\right]_{\mathrm{Sp}}+\left[2^{2}\right]_{\mathrm{Sp}}+3\left[1^{2}\right]_{\mathrm{Sp}}+2[0]_{\mathrm{Sp}}
\end{aligned}
$$

where $[0]_{\text {Sp }}$ denotes the trivial representation. For large $k$, we used the computer software $\mathrm{LiE}$ to obtain the irreducible decompositions. However, because of the memory problem we could obtain the GL-irreducible decomposition of $\wedge^{k}\left[1^{3}\right]_{\text {GL }}$ only up to $k=6$ or so. To overcome this difficulty, we used the Adams operations $\psi^{k}(k=1,2, \ldots)$ which satisfy the identity

$$
\wedge^{k} V=\frac{1}{k}\left(\wedge^{k-1} V \otimes V-\wedge^{k-2} V \otimes \psi^{2}(V)+\cdots+(-1)^{k-1} \psi^{k}(V)\right)
$$

on any representation $V$. It turns out that the computer computation of the Adams operation is much easier than that of the exterior powers. By utilizing this merit of the Adams operations, we have determined so far the GL-irreducible decomposition of $\wedge^{k}\left[1^{3}\right]_{\mathrm{GL}}$ for $k \leq 10$ and the Sp-irreducible decomposition of $\wedge^{k}\left[1^{3}\right]_{\mathrm{GL}}$ for $k \leq 9$. As for the computation of the dimensions of the Sp-invariant subspaces of GL-modules with the form $\wedge^{k}\left[1^{3}\right]_{\mathrm{GL}} \otimes V$ for certain $V$, we can go further up to $k=16$ or so. Here we express $\wedge^{k}\left[1^{3}\right]_{\mathrm{GL}}(k=10,11, \ldots)$ in terms of $\wedge^{k}\left[1^{3}\right]_{\mathrm{GL}}(k=1,2, \ldots, 9)$ and $\psi^{k}\left[1^{3}\right]_{\mathrm{GL}}(k=1,2, \ldots)$ and apply the preceding methods. The coefficients of this expression are complicated rational numbers rather than the integers. From this fact, we obtain an extra merit of this method. Namely, we can check the accuracy of the computation just by confirming the answer to be an integer because it is most likely that any small mistake in the computation would force that the output is not an integer. 
Method (VI) (Counting the number of graphs with a prescribed type)

In [49], a certain linear mapping

$\mathbb{Q}\langle$ isomorphism class of trivalent graph with $2 k$-verticies $\rangle \rightarrow\left(\wedge^{2 k}\left[1^{3}\right]_{\mathrm{GL}}\right)^{\mathrm{Sp}}$

was introduced by making use of a classical result of Weyl, which is an isomorphism in the stable range. Here the left hand side denotes the vector space generated by the isomorphism classes of trivalent graphs with $2 k$ vertices where we allow a trivalent graph to have multi-edges and/or loops. In the theory of enumeration of graphs, the numbers of such trivalent graphs are known for $k \leq 16$ by making use of the result of Read [63].

Method (VII) (Checking the accuracy of computations)

We have adopted a few checking procedure to confirm the accuracy of our computations. As for the irreducible decompositions, we have checked that the dimension of the resulting decomposition coincides with that of the original module by applying the Weyl character formula. As for the dimension counting of various Sp-invariant subspaces, we carried out multiple different ways of computations and checked that the answers coincide with each other.

In short, our strategy is a mixture of theoretical considerations and computer computations. By combining the above Methods (I)-(VII) in various ways, we made explicit computer computations the results of which will be given in the following three sections.

\section{THE CASE OF $\mathfrak{c}_{g}$ AND THE GRAPH HOMOLOGY AS WELL AS TRANSVERSELY SYMPLECTIC FOLIATIONS}

First we consider the commutative case. From the point of view of explicit computations, this case of $\mathfrak{c}_{g}$ is the simplest among the three Lie algebras because each piece $\mathfrak{c}_{g}(k) \cong S^{k+2} H_{\mathbb{Q}}$ is a single irreducible $\operatorname{Sp}(2 g, \mathbb{Q})$-module. However, its stable (co)homology is far from being well understood and there are big mysteries here. Before describing them, the result of our computation for this case is depicted in Table 1 .

Here $C_{k}$ of the weight $w$ part denotes

$$
\lim _{g \rightarrow \infty} \operatorname{dim}\left(\bigoplus_{\substack{i_{1}+i_{2}+\cdots+i_{w}=k \\ i_{1}+2 i_{2}+\cdots+w i_{w}=w}} \wedge^{i_{1}}\left(S^{3} H_{\mathbb{Q}}\right) \otimes \wedge^{i_{2}}\left(S^{4} H_{\mathbb{Q}}\right) \otimes \cdots \otimes \wedge^{i_{w}}\left(S^{w+2} H_{\mathbb{Q}}\right)\right)^{\mathrm{Sp}}
$$

so that we have a finite dimensional chain complex $0 \rightarrow C_{w} \rightarrow \cdots \rightarrow C_{1} \rightarrow 0$. Also $\chi$ denotes the Euler characteristic of this chain complex, namely the weight $w$ part of the Sp-invariant stable homology $H_{*}\left(\mathfrak{c}_{\infty}^{+}\right)_{w}^{\mathrm{Sp}}$.

Proof of Theorem 1.1(i). This follows from Table1. 
TABLE 1. Case of $\mathfrak{c}_{g}$

\begin{tabular}{|c|c|c|c|c|c|c|c|c|c|c|}
\hline$w$ & 2 & 4 & 6 & 8 & 10 & 12 & 14 & 16 & 18 & 20 \\
\hline$C_{1}$ & 0 & 0 & 0 & 0 & 0 & 0 & 0 & 0 & 0 & 0 \\
\hline$C_{2}$ & 1 & 0 & 1 & 0 & 1 & 0 & 1 & 0 & 1 & 0 \\
\hline$C_{3}$ & & 1 & 1 & 4 & 3 & 8 & 6 & 12 & 10 & 17 \\
\hline$C_{4}$ & & 3 & 0 & 16 & 20 & 63 & 78 & 164 & 205 & 355 \\
\hline$C_{5}$ & & & 4 & 20 & 112 & 271 & 748 & 1484 & 3103 & 5447 \\
\hline$C_{6}$ & & & 7 & 15 & 269 & 1013 & 3964 & 11047 & 29423 & 67611 \\
\hline$C_{7}$ & & & & 25 & 310 & 2784 & 14034 & 59153 & 200982 & 613281 \\
\hline$C_{8}$ & & & & 24 & 223 & 4690 & 36530 & 220693 & 1023318 & 4068707 \\
\hline$C_{9}$ & & & & & 166 & 4683 & 68504 & 592111 & 3862954 & 20226716 \\
\hline$C_{10}$ & & & & & 86 & 2963 & 87552 & 1167459 & 10828229 & 76399055 \\
\hline$C_{11}$ & & & & & & 1395 & 73358 & 1682134 & 22709573 & 220634704 \\
\hline$C_{12}$ & & & & & & 426 & 39797 & 1727415 & 35748802 & 488935936 \\
\hline$C_{13}$ & & & & & & & 13984 & 1221607 & 41935536 & 832479480 \\
\hline$C_{14}$ & & & & & & & 2732 & 570419 & 35952084 & 1085617203 \\
\hline$C_{15}$ & & & & & & & & 164365 & 21796235 & 1073488879 \\
\hline$C_{16}$ & & & & & & & & 23701 & 8867266 & 789223120 \\
\hline$C_{17}$ & & & & & & & & & 2199842 & 417233525 \\
\hline$C_{18}$ & & & & & & & & & 258951 & 149905889 \\
\hline$C_{19}$ & & & & & & & & & & 32900910 \\
\hline$C_{20}$ & & & & & & & & & & 3365151 \\
\hline total & 1 & 4 & 13 & 104 & 1190 & 18296 & 341288 & 7441764 & 185416514 & 5195165986 \\
\hline$\chi$ & 1 & 2 & 3 & 6 & 8 & 14 & 20 & 32 & 44 & 68 \\
\hline
\end{tabular}

Let $G_{*}^{(n)}(n \geq 2)$ be the graph complex defined by Kontsevich, which is a chain complex of dimension $2 n-2$.

Theorem 5.1 (Kontsevich [38,39]). For any $k \geq 1$ and $n \geq 1$, there exists an isomorphism

$$
P H_{k}\left(\mathfrak{c}_{\infty}^{+}\right)_{2 n}^{\mathrm{Sp}} \cong H_{k}\left(G_{*}^{(n+1)}\right) .
$$

Proposition 5.2. The weight generating function, denoted by $c(t)$, for the Sp-invariant stable homology group $H_{*}\left(\mathfrak{c}_{\infty}^{+}\right)^{\mathrm{Sp}}$ is given by

$$
c(t)=\prod_{n=2}^{\infty}\left(1-t^{2 n-2}\right)^{-e\left(G_{*}^{(n)}\right)}
$$

where $e\left(G_{*}^{(n)}\right)$ denotes the Euler characteristic of $H_{*}\left(G_{*}^{(n)}\right)$.

Proof. This follows from Theorem 5.1 and Proposition 2.4.

Proof of Theorem 1.2(i). By Theorem1.1(i), we see that the weight generating function

$$
c(t)=\sum_{w=0}^{\infty} \chi\left(H_{*}\left(\mathfrak{c}_{\infty}^{+}\right)_{w}^{\mathrm{Sp}}\right) t^{w}
$$


for $H_{*}\left(\mathfrak{c}_{\infty}^{+}\right)^{\mathrm{Sp}}$, up to weight 20 , is given by

$$
c(t)=1+t^{2}+2 t^{4}+3 t^{6}+6 t^{8}+8 t^{10}+14 t^{12}+20 t^{14}+32 t^{16}+44 t^{18}+68 t^{20}+\cdots .
$$

By applying Proposition 2.6, we can inductively determine the integral Euler characteristics of the primitive parts, namely $e\left(G_{*}^{(n)}\right)$. If we put

$$
\begin{aligned}
\bar{c}(t)=\left(1-t^{2}\right)^{-1} & \left(1-t^{4}\right)^{-1}\left(1-t^{6}\right)^{-1}\left(1-t^{8}\right)^{-2} \\
& \left(1-t^{10}\right)^{-1}\left(1-t^{12}\right)^{-2}\left(1-t^{14}\right)^{-2}\left(1-t^{16}\right)^{-2}\left(1-t^{18}\right)^{-1}\left(1-t^{20}\right)^{-3},
\end{aligned}
$$

then we see that

$$
c(t)-\bar{c}(t) \equiv 0 \bmod t^{21} .
$$

By Proposition 5.2, we can now conclude that $e\left(G_{*}^{(n)}\right)=1,1,1,2,1,2,2,2,1,3$ for $n=$ $2,3, \ldots, 11$, respectively. The result is depicted in the fourth row of Table 2 .

TABLE 2. Numbers of new generators for $H_{*}\left(\mathfrak{c}_{\infty}^{+}\right)_{w}^{\mathrm{Sp}}$

\begin{tabular}{|c|l|l|l|l|r|r|r|r|r|r|}
\hline$w$ & 2 & 4 & 6 & 8 & 10 & 12 & 14 & 16 & 18 & 20 \\
\hline$\chi$ & 1 & 2 & 3 & 6 & 8 & 14 & 20 & 32 & 44 & 68 \\
\hline$\chi$ of lower terms & 0 & 1 & 2 & 4 & 7 & 12 & 18 & 30 & 43 & 65 \\
\hline$\chi$ of primitive part & 1 & 1 & 1 & 2 & 1 & 2 & 2 & 2 & 1 & 3 \\
\hline $\operatorname{dim} \mathcal{A}(\emptyset)^{(w)}$ & 1 & 2 & 3 & 6 & 9 & 16 & 25 & 42 & 65 & 105 \\
\hline generators for $\mathcal{A}(\emptyset)$ & 1 & 1 & 1 & 2 & 2 & 3 & 4 & 5 & 6 & 8 \\
\hline$\chi$ of primitive part of $\mathcal{E}$ & 0 & 0 & 0 & 0 & -1 & -1 & -2 & -3 & -5 & -5 \\
\hline
\end{tabular}

As was already mentioned in the introduction, there are deep connections of this case with two important subjects in topology. Namely the theory of finite type invariants of homology 3-spheres as well as 3-manifolds and the theory of characteristic classes of transversely symplectic foliations.

In [22] (Theorem 2), Garoufalidis and Nakamura proved the following beautiful result. Stably there exists an isomorphism

$$
\mathcal{A}(\emptyset) \cong\left(\wedge^{*}\left(S^{3} H_{\mathbb{Q}}\right) /\left([4]_{\mathrm{Sp}}\right)\right)^{\mathrm{Sp}}
$$

of graded algebras. Here $[4]_{\mathrm{Sp}}=S^{4} H_{\mathbb{Q}} \subset \wedge^{2}\left(S^{3} H_{\mathbb{Q}}\right)$ denotes a certain summand and $\left([4]_{\mathrm{Sp}}\right)$ denotes the ideal of $\wedge^{*}\left(S^{3} H_{\mathbb{Q}}\right)$ generated by it. This result is closely related to the following well-known fact which shows that the top homology groups of the graph complexes correspond to the finite type invariants of 3-manifolds.

Proposition 5.3. There exists an isomorphism

$$
H_{2 n-2}\left(G_{*}^{(n)}\right) \cong \mathcal{A}(\emptyset)_{\text {conn }}^{(2 n-2)} \text {. }
$$

Here we derive this fact from the above theorem of Garoufalidis and Nakamura, in the framework of this paper, just for completeness. In the chain complex computing $H_{*}\left(\mathfrak{c}_{g}^{+}\right)_{2}$ (the weight 2 part), the boundary operator is the Poisson bracket

$$
\wedge^{2}\left(S^{3} H_{\mathbb{Q}}\right) \stackrel{\partial}{\longrightarrow} S^{4} H_{\mathbb{Q}}
$$


which is easily seen to be surjective. Passing to the dual, the cochain complex computing $H_{c}^{*}\left(\widehat{\mathfrak{c}}_{g}^{+}\right)_{2}$ is

$$
S^{4} H_{\mathbb{Q}} \stackrel{\delta}{\longrightarrow} \wedge^{2}\left(S^{3} H_{\mathbb{Q}}\right)
$$

which is easily seen to be injective. Here recall that any finite dimensional Sp-module is canonically isomorphic to its dual module. Since the multiplicity of $S^{4} H_{\mathbb{Q}}$ in $\wedge^{2}\left(S^{3} H_{\mathbb{Q}}\right)$ is one as already pointed out in [22], this is the same summand as above. By the definition of the Lie algebra cohomology, we can now conclude that the final part of the cochain complex computing $H_{c}^{*}\left(\widehat{\mathfrak{c}}_{g}^{+}\right)_{2 k}^{\mathrm{Sp}}$ is

$$
\cdots \rightarrow\left(S^{4} H_{\mathbb{Q}} \otimes \wedge^{2 k-2}\left(S^{3} H_{\mathbb{Q}}\right)\right)^{\mathrm{Sp}} \stackrel{\wedge(\delta \otimes \mathrm{id})}{\longrightarrow}\left(\wedge^{2 k}\left(S^{3} H_{\mathbb{Q}}\right)\right)^{\mathrm{Sp}} \rightarrow 0
$$

where the last non-trivial homomorphism can be identified with $\delta \otimes$ id followed by the wedge product $\wedge$. This is because $\delta(\alpha \wedge \beta)=(\delta \alpha) \wedge \beta+(-1)^{\operatorname{deg} \alpha} \alpha \wedge(\delta \beta)$ in general and $\delta \beta=0$ for any $\beta \in S^{3} H_{\mathbb{Q}}$ in the present case. Now the top cohomology group $H_{c}^{2 k}\left(\widehat{\mathfrak{c}}_{g}^{+}\right)_{2 k}^{\mathrm{Sp}}$ is the cokernel of the above homomorphism and clearly the image of $\wedge(\delta \otimes \mathrm{id})$ coincides with the ideal $\left(S^{4} H_{\mathbb{Q}}\right)$. One can now apply the result of Garoufalidis and Nakamura above to obtain an isomorphism

$$
H_{c}^{2 k}\left(\widehat{\mathfrak{c}}_{g}^{+}\right)_{2 k}^{\mathrm{Sp}} \cong \mathcal{A}(\emptyset)^{(2 k)} .
$$

By passing to the dual, this yields an isomorphism

$$
H_{2 k}\left(\mathfrak{c}_{g}^{+}\right)_{2 k}^{\mathrm{Sp}} \cong \mathcal{A}(\emptyset)^{(2 k)}
$$

of the top homology group. Proposition 5.3 follows from this by restricting to the primitive part.

We can now conclude that there exists an injective homomorphism

$$
\mathcal{A}(\emptyset) \rightarrow H_{*}\left(\mathfrak{c}_{\infty}^{+}\right)^{\mathrm{Sp}}
$$

and let us consider $\mathcal{A}(\emptyset)$ as a subalgebra of $H_{*}\left(\mathfrak{c}_{\infty}^{+}\right)^{\mathrm{Sp}}$.

Definition 5.4. Let $\mathcal{A}^{+}(\emptyset)$ denote the subalgebra of $\mathcal{A}(\emptyset)$ consisting of all the elements with positive degrees and let $\mathcal{I}\left(\mathcal{A}^{+}(\emptyset)\right)$ denote the ideal of $H_{*}\left(\mathfrak{c}_{\infty}^{+}\right)^{\text {Sp }}$ generated by $\mathcal{A}^{+}(\emptyset)$. Now set

$$
\mathcal{E}=H_{*}\left(\mathfrak{c}_{\infty}^{+}\right)^{\mathrm{Sp}} / \mathcal{I}\left(\mathcal{A}^{+}(\emptyset)\right)
$$

which is a free graded commutative algebra with respect to the degree. It is also equipped with the second grading induced by the weights.

By the definition, clearly we have an isomorphism

$$
H_{*}\left(\mathfrak{c}_{\infty}^{+}\right)^{\mathrm{Sp}} \cong \mathcal{A}(\emptyset) \otimes \mathcal{E}
$$

of bigraded algebras.

Proof of Theorem 1.3. Although the structure of the polynomial algebra $\mathcal{A}(\emptyset)$ is far from being understood, it is known that the numbers of generators for this algebra are $1,1,1,2,2,3,4,5,6,8,9$ for degrees $w=2,4, \ldots, 22$ (see [60]) and the generating function of this algebra is

$\phi(t)=1+t^{2}+2 t^{4}+3 t^{6}+6 t^{8}+9 t^{10}+16 t^{12}+25 t^{14}+42 t^{16}+65 t^{18}+105 t^{20}+161 t^{22}+\cdots$. 
We write these values in the fifth and the sixth rows of Table 2. Then by subtracting the sixth row from the fourth row of Table 2, we can determine the first several terms of the weight generating function for the bigraded algebra $\mathcal{E}$ to be

$$
e(t)=1-t^{10}-t^{12}-2 t^{14}-3 t^{16}-5 t^{18}-5 t^{20}+\cdots .
$$

Of course we should have the identity $c(t)=\phi(t) e(t)$ which is easy to check. This completes the proof.

In the framework of our bigraded algebra $\mathcal{E}$, the results of Gerlits [25] (Theorem 4.1.) as well as Conant, Gerlits and Vogtmann [10] (Theorem 5.1.) can be described as follows. Namely, $\mathcal{E}_{w}=0$ for all $w=2, \ldots, 8$ and $\mathcal{E}_{10} \cong \mathbb{Q}, \mathcal{E}_{12} \cong \mathbb{Q}$ are spanned by certain elements in $\mathrm{PH}_{7}\left(\mathfrak{c}_{\infty}^{+}\right)_{10}^{\mathrm{Sp}}$ and $P H_{9}\left(\mathfrak{c}_{\infty}^{+}\right)_{12}^{\mathrm{Sp}}$, respectively.

Conjecture 5.5. The free graded algebra $\mathcal{E}$ is infinitely generated. Furthermore there exist infinitely many generators with odd degrees.

Problem 5.6. Construct explicit cycles lying in $P \mathcal{E}$.

Next we describe the connection of the commutative case with the theory of characteristic classes of transversely symplectic foliations. Let $\mathfrak{h a m}_{2 g}$ denote the Lie algebra consisting of all the formal Hamiltonian vector fields on $\mathbb{R}^{2 g}$ with respect to the standard symplectic form. In [40], Kontsevich considered two Lie subalgebras

$$
\mathfrak{h a m} \mathfrak{m}_{2 g}^{1} \subset \mathfrak{h a m} \mathfrak{m}_{2 g}^{0} \subset \mathfrak{h a m} \mathfrak{m}_{2 g}
$$

where $\mathfrak{h a m}_{2 g}^{0}$ and $\mathfrak{h a m} \mathfrak{a m}_{2 g}^{1}$ denote the Lie subalgebra consisting of formal Hamiltonian vector fields without constant terms and without constant as well as linear terms, respectively. He gave a geometric meaning to the Gelfand-Fuks cohomology

$$
H_{G F}^{*}\left(\mathfrak{h a m} \mathfrak{m}_{2 g}^{0}, \operatorname{Sp}(2 g, \mathbb{R})\right) \cong H_{G F}^{*}\left(\mathfrak{h a m} \mathfrak{m}_{2 g}^{1}\right)^{\mathrm{Sp}}
$$

as follows. Let $\mathcal{F}$ be a transversely symplectic foliation on a smooth manifold $M$ of codimension $2 g$ and let $H_{\mathcal{F}}^{*}(M)$ be the associated foliated cohomology group. Then he constructed a homomorphism

$$
H_{G F}^{*}\left(\mathfrak{h a m} \mathfrak{m}_{2 g}^{1}\right)^{\mathrm{Sp}} \rightarrow H_{\mathcal{F}}^{*}(M)
$$

Now it is easy to see that the Lie algebras $\mathfrak{h a m}_{2 g}^{0}, \mathfrak{h a m} \mathfrak{a m}_{2 g}^{1}$ are nothing other than the completions of $\mathfrak{c}_{g} \otimes \mathbb{R}, \mathfrak{c}_{g}^{+} \otimes \mathbb{R}$ with respect to the natural gradings so that we can write

$$
\mathfrak{h a m} \mathfrak{m}_{2 g}^{0}=\widehat{\mathfrak{c}}_{g} \otimes \mathbb{R}, \quad \mathfrak{h} \mathfrak{a m}_{2 g}^{1}=\widehat{\mathfrak{c}}_{g}^{+} \otimes \mathbb{R} .
$$

It follows that we have a homomorphism

$$
H_{c}^{*}\left(\widehat{\mathfrak{c}}_{g}^{+}\right)^{\mathrm{Sp}} \otimes \mathbb{R} \cong H_{G F}^{*}\left(\mathfrak{h a m}_{2 g}^{1}\right)^{\mathrm{Sp}} \rightarrow H_{\mathcal{F}}^{*}(M)
$$

for any transversely symplectic foliation $(M, \mathcal{F})$. Let $\mathfrak{c}_{g}^{+} \rightarrow \mathfrak{c}_{g}(1)=S^{3} H_{\mathbb{Q}}$ be the projection. Then the composition

$$
H^{*}\left(S^{3} H_{\mathbb{Q}}\right)^{\mathrm{Sp}} \rightarrow H_{c}^{*}\left(\widehat{\mathfrak{c}}_{g}^{+}\right)^{\mathrm{Sp}} \otimes \mathbb{R} \rightarrow H_{\mathcal{F}}^{*}(M)
$$

produces the usual leaf cohomology classes in the sense that they are expressed by differential forms involving only the connection form and the curvature form including 
the Pontrjagin forms. It follows that our bigraded algebra $\mathcal{E}$ can be interpreted as the dual of the space of all the exotic stable leaf cohomology classes, as already mentioned in the introduction.

Problem 5.7. Study the geometric meaning of the classes in $\mathcal{E}$ in the context of universal characteristic classes for odd dimensional manifold bundles as well as characteristic classes for transversely symplectic foliations.

6. THE CASE OF $\mathfrak{h}_{g, 1}$ AND THE OUTER AUTOMORPHISM GROUPS OF FREE GROUPS

Next we consider the Lie case. The result of our computation for this case is depicted in Table 3 ,

TABLE 3. Case of $\mathfrak{h}_{g, 1}$

\begin{tabular}{|c|c|c|c|c|c|c|c|c|c|}
\hline$w$ & 2 & 4 & 6 & 8 & 10 & 12 & 14 & 16 & 18 \\
\hline$C_{1}$ & 1 & 0 & 5 & 3 & 108 & 650 & 8817 & 111148 & 1729657 \\
\hline$C_{2}$ & 2 & 0 & 10 & 66 & 580 & 6621 & 84756 & 1281253 & 21671535 \\
\hline$C_{3}$ & & 6 & 7 & 239 & 1928 & 29219 & 424358 & 7286710 & 137344661 \\
\hline$C_{4}$ & & 8 & 16 & 342 & 4946 & 78443 & 1400274 & 27097563 & 575398310 \\
\hline$C_{5}$ & & & 41 & 293 & 8375 & 152310 & 3289532 & 73457788 & 1766236662 \\
\hline$C_{6}$ & & & 31 & 287 & 8887 & 227058 & 5780112 & 152604335 & 4190265424 \\
\hline$C_{7}$ & & & & 294 & 6536 & 254063 & 7885801 & 249166200 & 7923956179 \\
\hline$C_{8}$ & & & & 140 & 4175 & 206753 & 8491679 & 324662115 & 12158481555 \\
\hline$C_{9}$ & & & & & 2353 & 123990 & 7160718 & 340745360 & 15284159637 \\
\hline$C_{10}$ & & & & & 722 & 58302 & 4634679 & 288478215 & 15809478819 \\
\hline$C_{11}$ & & & & & & 21368 & 2269538 & 195270880 & 13456339409 \\
\hline$C_{12}$ & & & & & & 4439 & 836620 & 103755671 & 7824793027 \\
\hline$C_{13}$ & & & & & & & 221987 & 42207231 & 5290518430 \\
\hline$C_{14}$ & & & & & & & 32654 & 12701040 & 2368530727 \\
\hline$C_{15}$ & & & & & & & & 2624381 & 816469677 \\
\hline$C_{16}$ & & & & & & & & 289519 & 206593733 \\
\hline$C_{17}$ & & & & & & & & & 34966981 \\
\hline$C_{18}$ & & & & & & & & & 3054067 \\
\hline total & 3 & 14 & 110 & 1664 & 38610 & 1163216 & 42521525 & 1821739409 & 89423442490 \\
\hline$\chi$ & 1 & 2 & 4 & 6 & 10 & 16 & 23 & 13 & -96 \\
\hline
\end{tabular}

Proof of Theorem 1.1(ii). This follows from Table 3 .

Theorem 6.1 (Kontsevich [38, 39]). For any $k \geq 1$ and $n \geq 1$, there exists an isomorphism

$$
P H_{k}\left(\mathfrak{h}_{\infty, 1}^{+}\right)_{2 n}^{\mathrm{Sp}} \cong H^{2 n-k}\left(\text { Out } F_{n+1} ; \mathbb{Q}\right) \text {. }
$$

Proposition 6.2. The weight generating function, denoted by $h(t)$, for the Sp-invariant stable homology group $H_{*}\left(\mathfrak{h}_{\infty, 1}^{+}\right)^{\mathrm{Sp}}$ is given by

$$
h(t)=\prod_{n=2}^{\infty}\left(1-t^{2 n-2}\right)^{-e\left(\text { Out } F_{n}\right)}
$$


where $e\left(\right.$ Out $\left.F_{n}\right)$ denotes the integral Euler characteristic of Out $F_{n}$.

Proof. This follows from Theorem 6.1 and Proposition 2.4.

Proof of Theorem 1.2(ii). By Theorem 1.1(ii), we see that the weight generating function

$$
h(t)=\sum_{w=0}^{\infty} \chi\left(H_{*}\left(\mathfrak{h}_{\infty, 1}^{+}\right)_{w}^{\mathrm{Sp}}\right) t^{w}
$$

for $H_{*}\left(\mathfrak{h}_{\infty, 1}^{+}\right)^{\mathrm{Sp}}$, up to weight 18 , is given by

$$
h(t)=1+t^{2}+2 t^{4}+4 t^{6}+6 t^{8}+10 t^{10}+16 t^{12}+23 t^{14}+13 t^{16}-96 t^{18}+\cdots .
$$

By applying Proposition 2.6, we can inductively determine the Euler characteristics of the primitive parts, namely $e\left(\operatorname{Out} F_{n}\right)$. If we put

$$
\begin{aligned}
\bar{h}(t)=\left(1-t^{2}\right)^{-1}\left(1-t^{4}\right)^{-1} & \left(1-t^{6}\right)^{-2}\left(1-t^{8}\right)^{-1} \\
& \left(1-t^{10}\right)^{-2}\left(1-t^{12}\right)^{-1}\left(1-t^{14}\right)^{-1}\left(1-t^{16}\right)^{21}\left(1-t^{18}\right)^{124}
\end{aligned}
$$

then we see that

$$
h(t)-\bar{h}(t) \equiv 0 \bmod t^{19} .
$$

By Proposition 6.2, we can now conclude that $e\left(\right.$ Out $\left.F_{n}\right)=1,1,2,1,2,1,1,-21,-124$ for $n=2,3, \ldots, 10$, respectively. The result is depicted in the fourth row of Table 4 .

TABLE 4. Numbers of new generators for $H_{*}\left(\mathfrak{h}_{\infty, 1}^{+}\right)_{w}^{\mathrm{Sp}}$

\begin{tabular}{|c|r|r|r|r|r|r|r|r|r|}
\hline$w$ & 2 & 4 & 6 & 8 & 10 & 12 & 14 & 16 & 18 \\
\hline$\chi$ & 1 & 2 & 4 & 6 & 10 & 16 & 23 & 13 & -96 \\
\hline$\chi$ of lower terms & 0 & 1 & 2 & 5 & 8 & 15 & 22 & 34 & 28 \\
\hline$\chi$ of primitive part & 1 & 1 & 2 & 1 & 2 & 1 & 1 & -21 & -124 \\
\hline
\end{tabular}

Thus we see that there are many odd dimensional non-trivial rational cohomology classes of Out $F_{9}$ as well as Out $F_{10}$. Before this result, very few results have been known about the rational cohomology group of Out $F_{n}$. As for the cases $n \leq 6$, by the works of Hatcher and Vogtmann [35] as well as Ohashi [58], the only non-trivial cohomology groups are $H^{4}\left(\right.$ Out $\left.F_{4} ; \mathbb{Q}\right) \cong \mathbb{Q}$ and $H^{8}\left(\right.$ Out $\left.F_{6} ; \mathbb{Q}\right) \cong \mathbb{Q}$. On the other hand, by making use of the trace maps introduced in [48] which give a large abelian quotient of $\mathfrak{h}_{g, 1}^{+}$, the first named author defined many rational homology classes of Out $F_{n}$ in [50][51], the most important classes being a series of homology classes

$$
\mu_{k} \in H_{4 k}\left(\text { Out } F_{2 k+2} ; \mathbb{Q}\right) \quad(k=1,2, \ldots) .
$$

It was conjectured in [50] that these will be all non-trivial. However, at present only the first three classes are known to be non-trivial, $\mu_{1}$ in [50], $\mu_{2}$ by Conant and Vogtmann [12] and $\mu_{3}$ by Gray [31]. Conant and Vogtmann also gave a geometric construction of many homology classes in the framework of the Outer Space of Culler and Vogtmann [14].

As already mentioned in the introduction, recently, Conant, Kassabov and Vogtmann [11] proved a remarkable result about the structure of $\mathfrak{h}_{g, 1}$. They found a deep 
connection with the theory of elliptic modular forms by which they show the existence of a large new abelianization beyond the trace maps. In particular, they defined many new cohomology classes in $H_{c}^{2}\left(\widehat{\mathfrak{h}}_{\infty, 1}^{+}\right)_{2 w}^{\mathrm{Sp}}$ whenever the dimension of the cusp forms of some weight $w$ is larger than 1 , the first one being $w=24$. These classes then produce, by Theorem 6.1, rational homology classes of Out $F_{n}$ the first of which lies in $H_{46}\left(\right.$ Out $\left.F_{25} ; \mathbb{Q}\right)$.

Now we go back to the case of Out $F_{7}$ which is the unknown case with the smallest rank. By our result Theorem 1.2 (ii), the Euler characteristic of this group is 1 and it is an interesting problem to determine whether the rational cohomology group of this group is trivial or not. See Problem 9.4 for this. Next we consider Out $F_{8}$. Again by Theorem 1.2 (ii), $e\left(\right.$ Out $\left.F_{8}\right)=1$. On the other hand, Gray [31] proved that $\mu_{3} \neq 0 \in H_{12}\left(\right.$ Out $\left.F_{8} ; \mathbb{Q}\right)$. It follows that there exists at least one odd dimensional rational homology class. Here we propose a candidate of such a class in the following proposition $\left(\gamma_{1} \in H_{11}\right.$ (Out $\left.F_{8} ; \mathbb{Q}\right)$ is our candidate). For this, we use the summands $H_{1}\left(\mathfrak{h}_{\infty, 1}^{+}\right)_{2 k+4} \supset[2 k+1,1]_{\text {Sp }}(k=1,2, \ldots)$ which are part of the new abelianizations found by Conant, Kassabov and Vogtmann cited above. By an explicit computation motivated by their result, we have proved that $H_{1}\left(\mathfrak{h}_{g, 1}^{+}\right)_{6} \cong[31]_{\mathrm{Sp}}$.

Proposition 6.3. For any $k \geq 1$, we have an isomorphism

$$
\left([31]_{\mathrm{Sp}} \otimes[2 k+1]_{\mathrm{Sp}} \otimes[2 k+3]_{\mathrm{Sp}}\right)^{\mathrm{Sp}} \cong \mathbb{Q}
$$

so that we obtain a series of (co)homology classes

$$
\gamma_{k} \in P H_{c}^{3}\left(\widehat{\mathfrak{h}}_{\infty, 1}\right){ }_{4 k+10}^{\mathrm{Sp}} \stackrel{\text { Kontsevich }}{\cong} H_{4 k+7}\left(\text { Out } F_{2 k+6} ; \mathbb{Q}\right) \quad(k=1,2, \ldots) .
$$

Proof. By the Littlewood-Richardson rule, it is easy to see that

$$
[2 k+1]_{\mathrm{Sp}} \otimes[2 k+3]_{\mathrm{Sp}} \cong[4 k+4]_{\mathrm{GL}} \oplus[4 k+3,1]_{\mathrm{GL}} \oplus \cdots \oplus[2 k+3,2 k+1]_{\mathrm{GL}} .
$$

On the other hand, among the Sp-irreducible decompositions of the GL-irreducible summands on the right hand side, only the last one $[2 k+3,2 k+1]_{\mathrm{GL}}$ contains $[31]_{\mathrm{Sp}}$ and the multiplicity is 1 . The claim follows.

Conjecture 6.4. These classes are all non-trivial. In particular, $H_{3}\left(\mathfrak{h}_{\infty, 1}\right)$ is infinite dimensional.

Also if we combine the trace components $[2 k+1]_{\mathrm{Sp}}$ with the new components $[2 \ell+$ $1,1]_{\mathrm{Sp}}, \ldots$, we obtain huge amount of (co)homology classes of $\mathfrak{h}_{\infty, 1}$.

Next we consider the problem of comparison between the rational and the integral Euler characteristics of Out $F_{n}$. The second row of Table 5 is taken from Smillie and Vogtmann [65] where we write the values to the second decimal places and the third row is our Theorem 1.2 (ii).

Problem 6.5. Study the relation between $\chi\left(\right.$ Out $\left.F_{n}\right)$ and $e\left(\right.$ Out $\left.F_{n}\right)$.

We refer to the book [17] edited by Farb, in particular Bridson and Vogtmann [5], as well as Farb [18] for various problems concerning Out $F_{n}$, mapping class groups, $\mathrm{GL}(n, \mathbb{Z})$ and other related groups. 
TABLE 5. $\chi$ versus $e$ for Out $F_{n}$

\begin{tabular}{|c|c|c|c|c|c|c|c|c|c|}
\hline$n$ & 2 & 3 & 4 & 5 & 6 & 7 & 8 & 9 & 10 \\
\hline$\chi$ & -0.04 & -0.02 & -0.02 & -0.06 & -0.20 & -0.87 & -4.58 & -28.52 & -205.83 \\
\hline$e$ & 1 & 1 & 2 & 1 & 2 & 1 & 1 & -21 & -124 \\
\hline
\end{tabular}

\section{THE CASE OF $\mathfrak{a}_{g}$ AND THE MODULI SPACES OF CURVES}

Finally we consider the case of $\mathfrak{a}_{g}$. From the point of view of computations, this case is the most heavy one among the three Lie algebras as can be seen by comparing the size of the numbers in the former two tables Tables 1 and 3 with the present one depicted in Table 6 .

As was already mentioned in Section 1 , in [56] we determine the values $\chi\left(H_{*}\left(\mathfrak{a}_{\infty}^{+}\right)_{w}^{\mathrm{Sp}}\right)$ for all $w \leq 500$ by a completely different argument which makes use of a formula of Gorsky [30] for the equivariant Euler characteristics of the moduli spaces of curves $\mathbf{M}_{g}^{m}$. We confirm that the two values for $w \leq 16$ are the same. We think that this coincidence serves as a strong evidence for the accuracy of our computations in the other two cases $\mathfrak{c}_{\infty}, \mathfrak{h}_{\infty, 1}$. We mention that the existence of the formula of Gorsky depends heavily on the fact that the totality of $\mathbf{M}_{g}^{m}$ for various $g$ and $m$ makes a beautiful unified world. It seems unlikely that similar formulas will be found in the other two cases, at least in a near future.

TABLE 6. Case of $\mathfrak{a}_{g}$

\begin{tabular}{|c|c|c|c|c|c|c|c|c|}
\hline$w$ & 2 & 4 & 6 & 8 & 10 & 12 & 14 & 16 \\
\hline$C_{1}$ & 1 & 2 & 17 & 88 & 897 & 9562 & 127071 & 1912970 \\
\hline$C_{2}$ & 3 & 8 & 111 & 1146 & 14735 & 212965 & 3483545 & 63522967 \\
\hline$C_{3}$ & & 18 & 289 & 5561 & 99285 & 1918401 & 39558275 & 880137499 \\
\hline$C_{4}$ & & 17 & 403 & 13653 & 366878 & 9590016 & 253890290 & 6966037951 \\
\hline$C_{5}$ & & & 320 & 19138 & 827528 & 30225682 & 1047033554 & 35904134757 \\
\hline$C_{6}$ & & & 124 & 15860 & 1193367 & 63894814 & 2967604968 & 129283963277 \\
\hline$C_{7}$ & & & & 7466 & 1111456 & 93211250 & 6001387476 & 339000966002 \\
\hline$C_{8}$ & & & & 1618 & 651577 & 94398768 & 8825700683 & 663520078156 \\
\hline$C_{9}$ & & & & & 220905 & 65356859 & 9484791225 & 982320832329 \\
\hline$C_{10}$ & & & & & 33564 & 29594121 & 7384704777 & 1104356533575 \\
\hline$C_{11}$ & & & & & & 7925093 & 4061192184 & 938047301852 \\
\hline$C_{12}$ & & & & & & 956263 & 1497800877 & 592700462357 \\
\hline$C_{13}$ & & & & & & & 332831365 & 270228006160 \\
\hline$C_{14}$ & & & & & & & 33736198 & 84077896041 \\
\hline$C_{15}$ & & & & & & & & 15987868100 \\
\hline$C_{16}$ & & & & & & & & 1402665692 \\
\hline total & 4 & 45 & 1264 & 64530 & 4520192 & 397293794 & 41933842488 & 5164742319685 \\
\hline$\chi$ & 2 & 5 & 12 & 24 & 50 & 100 & 188 & 347 \\
\hline
\end{tabular}

Proof of Theorem 1.1(iii). This follows from Table 6 , 
Theorem 7.1 (Kontsevich [38, 39]). For any $k \geq 1$ and $n \geq 1$, there exists an isomorphism

$$
P H_{k}\left(\mathfrak{a}_{\infty}^{+}\right)_{2 n}^{\mathrm{Sp}} \cong \bigoplus_{\substack{2 g-2+m=n \\ m>0}} H^{2 n-k}\left(\mathbf{M}_{g}^{m} ; \mathbb{Q}\right)^{\mathfrak{S}_{m}}
$$

Proposition 7.2. The weight generating function, denoted by $a(t)$, for the Sp-invariant stable homology group $H_{*}\left(\mathfrak{a}_{\infty}^{+}\right)^{\mathrm{Sp}}$ is given by

$$
a(t)=\prod_{n=1}^{\infty}\left(1-t^{2 n}\right)^{-a_{2 n}^{\mathrm{pr}}}
$$

where

$$
a_{2 n}^{\mathrm{pr}}=\sum_{\substack{2 g-2+m=n \\ m>0}} e\left(\mathbf{M}_{g}^{m} / \mathfrak{S}_{m}\right)
$$

Proof. Note that $H^{*}\left(\mathbf{M}_{g}^{m} ; \mathbb{Q}\right)^{\mathfrak{S}_{m}} \cong H^{*}\left(\mathbf{M}_{g}^{m} / \mathfrak{S}_{m} ; \mathbb{Q}\right)$. Our claim follows from Theorem 7.1 and Proposition 2.4

Proof of Theorem 1.2 (iii). By Theorem 1.1 (iii), we see that the weight generating function

$$
a(t)=\sum_{w=0}^{\infty} \chi\left(H_{*}\left(\mathfrak{a}_{\infty}^{+}\right)_{w}^{\mathrm{Sp}}\right) t^{w}
$$

for $H_{*}\left(\mathfrak{a}_{\infty}^{+}\right)^{\mathrm{Sp}}$, up to weight 16 , is given by

$$
a(t)=1+2 t^{2}+5 t^{4}+12 t^{6}+24 t^{8}+50 t^{10}+100 t^{12}+188 t^{14}+347 t^{16}+\cdots .
$$

By applying Proposition 2.6, we can inductively determine the Euler characteristics of the primitive parts, namely $e\left(\mathbf{M}_{g}^{m} / \mathfrak{S}_{m}\right)$. If we put

$$
\begin{aligned}
\bar{a}(t)=\left(1-t^{2}\right)^{-2}\left(1-t^{4}\right)^{-2} & \left(1-t^{6}\right)^{-4}\left(1-t^{8}\right)^{-2} \\
& \left(1-t^{10}\right)^{-6}\left(1-t^{12}\right)^{-6}\left(1-t^{14}\right)^{-6}\left(1-t^{16}\right)^{-1},
\end{aligned}
$$

then we see that

$$
a(t)-\bar{a}(t) \equiv 0 \bmod t^{17} .
$$

By Proposition 7.2, we can now conclude that $a_{2 n}^{\mathrm{pr}}=2,2,4,2,6,6,6,1$ for $n=1,2, \ldots, 8$, respectively. The result is depicted in the fourth row of Table 7 as well as the following proposition 7.3 .

TABLE 7. Numbers of new generators for $H_{*}\left(\mathfrak{a}_{\infty}^{+}\right)^{\mathrm{Sp}}$

\begin{tabular}{|c|r|r|r|r|r|r|r|r|}
\hline$w$ & 2 & 4 & 6 & 8 & 10 & 12 & 14 & 16 \\
\hline$\chi$ & 2 & 5 & 12 & 24 & 50 & 100 & 188 & 347 \\
\hline$\chi$ of lower terms & 0 & 3 & 8 & 22 & 44 & 94 & 182 & 346 \\
\hline$\chi$ of primitive part & 2 & 2 & 4 & 2 & 6 & 6 & 6 & 1 \\
\hline
\end{tabular}


Proposition 7.3. We have the following equalities.

(1) $e\left(\mathbf{M}_{0}^{3} / \mathfrak{S}_{3}\right)+e\left(\mathbf{M}_{1}^{1}\right)=2$

(2) $e\left(\mathbf{M}_{0}^{4} / \mathfrak{S}_{4}\right)+e\left(\mathbf{M}_{1}^{2} / \mathfrak{S}_{2}\right)=2$

(3) $e\left(\mathbf{M}_{0}^{5} / \mathfrak{S}_{5}\right)+e\left(\mathbf{M}_{1}^{3} / \mathfrak{S}_{3}\right)+e\left(\mathbf{M}_{2}^{1}\right)=4$

(4) $e\left(\mathbf{M}_{0}^{6} / \mathfrak{S}_{6}\right)+e\left(\mathbf{M}_{1}^{4} / \mathfrak{S}_{4}\right)+e\left(\mathbf{M}_{2}^{2} / \mathfrak{S}_{2}\right)=2$

(5) $e\left(\mathbf{M}_{0}^{7} / \mathfrak{S}_{7}\right)+e\left(\mathbf{M}_{1}^{5} / \mathfrak{S}_{5}\right)+e\left(\mathbf{M}_{2}^{3} / \mathfrak{S}_{3}\right)+e\left(\mathbf{M}_{3}^{1}\right)=6$

(6) $e\left(\mathbf{M}_{0}^{8} / \mathfrak{S}_{8}\right)+e\left(\mathbf{M}_{1}^{6} / \mathfrak{S}_{6}\right)+e\left(\mathbf{M}_{2}^{4} / \mathfrak{S}_{4}\right)+e\left(\mathbf{M}_{3}^{2} / \mathfrak{S}_{2}\right)=6$

(7) $e\left(\mathbf{M}_{0}^{9} / \mathfrak{S}_{9}\right)+e\left(\mathbf{M}_{1}^{7} / \mathfrak{S}_{7}\right)+e\left(\mathbf{M}_{2}^{5} / \mathfrak{S}_{5}\right)+e\left(\mathbf{M}_{3}^{3} / \mathfrak{S}_{3}\right)+e\left(\mathbf{M}_{4}^{1}\right)=6$

(8) $e\left(\mathbf{M}_{0}^{10} / \mathfrak{S}_{10}\right)+e\left(\mathbf{M}_{1}^{8} / \mathfrak{S}_{8}\right)+e\left(\mathbf{M}_{2}^{6} / \mathfrak{S}_{6}\right)+e\left(\mathbf{M}_{3}^{4} / \mathfrak{S}_{4}\right)+e\left(\mathbf{M}_{4}^{2} / \mathfrak{S}_{2}\right)=1$.

Now we check that our result above is consistent with the known results. By Getzler [27], $e\left(\mathbf{M}_{0}^{m} / \mathfrak{S}_{m}\right)=1$ for all $m \geq 3$. Also he determined the $\mathfrak{S}_{m}$-equivariant Serre polynomial of $\mathbf{M}_{1}^{m}$. In particular, he obtained the following formula

$$
\begin{aligned}
\sum_{m=1}^{\infty} e\left(\mathbf{M}_{1}^{m} / \mathfrak{S}_{m}\right) x^{m} & =\left(x+x^{2}+x^{3}\right) \frac{\left(1-x^{4}-2 x^{8}-x^{12}+x^{16}\right)}{\left(1-x^{8}\right)\left(1-x^{12}\right)} \\
& =x+x^{2}+x^{3}-x^{5}-x^{6}-x^{7}-x^{9}-x^{10}-x^{11}-x^{13}-x^{14}-\cdots
\end{aligned}
$$

so that $e\left(\mathbf{M}_{1}^{m} / \mathfrak{S}_{m}\right)=1,1,1,0,-1,-1,-1,0,-1$ for $m=1, \ldots, 9$. It is well known that $e\left(\mathbf{M}_{2}^{1}\right)=2$ and Getzler proved $e\left(\mathbf{M}_{2}^{2}\right)=1$. The fifth equality (5) (case of $w=10$ ) was first proved by Getzler and Kapranov [28], and then in [26] it was shown that $e\left(\mathbf{M}_{2}^{3} / \mathfrak{S}_{3}\right)=0$ by using the result of Looijenga [44] determining $H^{*}\left(\mathbf{M}_{3} ; \mathbb{Q}\right), H^{*}\left(\mathbf{M}_{3}^{1} ; \mathbb{Q}\right)$, especially $e\left(\mathbf{M}_{3}^{1}\right)=6$. Tommasi [66] (see also [67][68]) determined $H^{*}\left(\mathbf{M}_{4}, \mathbb{Q}\right.$ ) as well as the equivariant Hodge Euler characteristics of $\mathbf{M}_{2}^{4}$ and $\mathbf{M}_{3}^{2}$ and in particular $e\left(\mathbf{M}_{2}^{4} / \mathfrak{S}_{4}\right)=$ 1 and $e\left(\mathbf{M}_{3}^{2} / \mathfrak{S}_{2}\right)=5$. The sixth equality (6) is consistent with these results. Next we consider the seventh equality. By Harer and Zagier [34], $e\left(\mathbf{M}_{4}^{1}\right)=2$. Gorsky [29] (Theorem 2) extended the work of Getzler and obtained a formula for the $\mathfrak{S}_{m}$-equivariant Euler characteristic for $\mathbf{M}_{2}^{m}$. More precisely he obtained a formula for the generating function $\sum_{m=0}^{\infty} e^{\mathfrak{S}_{m}}\left(\mathbf{M}_{2}^{m}\right) t^{m}$ in terms of Newton's power sum polynomials. He then made a computer computation and determined $e^{\mathfrak{S}_{m}}\left(\mathbf{M}_{2}^{m}\right)$ explicitly for all $m \leq 4$ which coincide with the former results of Getzler and Tommasi cited above. By making use of our Method (I) and Method (V) described in Section 4, we extended Gorsky's computation to obtain closed formulas for the cases $5 \leq m \leq 35$. Here we describe the 
results for $m=5,6,7,8$.

$$
\begin{aligned}
e^{\mathfrak{S}_{5}}\left(\mathbf{M}_{2}^{5}\right) & =2[5]-2[32]+2[41] \\
e^{\mathfrak{S}_{6}}\left(\mathbf{M}_{2}^{6}\right) & =-2\left[3^{2}\right]+2[51]-3\left[2^{3}\right]-2[321]+2\left[41^{2}\right]+\left[2^{2} 1^{2}\right]-\left[21^{4}\right]-\left[1^{6}\right] \\
e^{\mathfrak{S}_{7}}\left(\mathbf{M}_{2}^{7}\right) & =-2[7]-2[43]+2[52]-2[61]-4\left[32^{2}\right]-2\left[3^{2} 1\right]+4[421]+2\left[51^{2}\right]- \\
& 2\left[2^{3} 1\right]+4\left[321^{2}\right]+2\left[41^{3}\right]-2\left[21^{5}\right]-2\left[1^{7}\right] \\
e^{\mathfrak{S}_{8}}\left(\mathbf{M}_{2}^{8}\right) & =-[8]-3\left[4^{2}\right]-4[53]+2[62]-3[71]-7\left[3^{2} 2\right]+4\left[42^{2}\right]-7[431]+2[521]- \\
& 7\left[61^{2}\right]-4\left[2^{4}\right]-3\left[32^{2} 1\right]-2\left[3^{2} 1^{2}\right]-\left[421^{2}\right]-3\left[51^{3}\right]-7\left[2^{3} 1^{2}\right]+2\left[41^{4}\right]- \\
& 3\left[2^{2} 1^{4}\right]-5\left[21^{6}\right]-3\left[1^{8}\right]
\end{aligned}
$$

It is amusing to calculate the dimensions of the above expression which give the integral Euler characteristics of $\mathbf{M}_{2}^{m}$. The results are $0,-24$ for $m=5,6$ and $(-1)^{m+1}(m+$ $1) ! / 240$ for $m \geq 7$ which coincide with the known values obtained by Harer and Zagier [34]. On the other hand, as for the coefficients of the trivial representation $[m]$, it can be shown that Gorsky's formula implies

$$
\begin{aligned}
\sum_{m=0}^{\infty} e & \left(\mathbf{M}_{2}^{m} / \mathfrak{S}_{m}\right) x^{m}=-\frac{1}{240}(1+x)^{-2}-\frac{1}{240}(1+x)^{6}\left(1+x^{2}\right)^{-4}+\frac{1}{12}(1+x)^{2}\left(1+x^{2}\right)^{-2} \\
& -\frac{1}{12}(1+x)^{4}\left(1+x^{3}\right)^{-2}-\frac{1}{8}(1+x)^{2}\left(1+x^{2}\right)^{2}\left(1+x^{4}\right)^{-2}+\frac{2}{5}(1+x)^{3}\left(1+x^{5}\right)^{-1} \\
& -\frac{1}{12}\left(1+x^{2}\right)^{2}\left(1+x^{3}\right)^{2}\left(1+x^{6}\right)^{-2}+\frac{1}{6}(1+x)^{2}\left(1+x^{2}\right)\left(1+x^{6}\right)^{-1} \\
& +\frac{1}{4}(1+x)^{2}\left(1+x^{4}\right)\left(1+x^{8}\right)^{-1}+\frac{2}{5}(1+x)\left(1+x^{2}\right)\left(1+x^{5}\right)\left(1+x^{10}\right)^{-1} \\
& =1+2 x+x^{2}+x^{4}+2 x^{5}-2 x^{7}-x^{8}-x^{10}+2 x^{12}+2 x^{13}-3 x^{14}+\cdots
\end{aligned}
$$

so that we find $e\left(\mathbf{M}_{2}^{m} / \mathfrak{S}_{m}\right)=2,0,-2,-1,0,-1,0,2,2,-3, \ldots$ for $m=5,6, \ldots, 14, \ldots$. By substituting these known values in the seventh equality $(7)$, we obtain $e\left(\mathbf{M}_{3}^{3} / \mathfrak{S}_{3}\right)=$ 2. This should be consistent with the work of Bergström [1] determining $H^{*}\left(\overline{\mathbf{M}}_{3}^{m} ; \mathbb{Q}\right)$ as well as $H^{*}\left(\mathbf{M}_{3}^{m} ; \mathbb{Q}\right)$ as an $\mathfrak{S}_{m}$-module for all $m \leq 5$, although the latter is not described explicitly. From the eighth equality (8) together with the above results, we conclude $e\left(\mathbf{M}_{3}^{4} / \mathfrak{S}_{4}\right)+e\left(\mathbf{M}_{4}^{2} / \mathfrak{S}_{2}\right)=0$. Now Gorsky [30] (Theorem 3) extended his own result cited above to obtain a formula for the equivariant Euler characteristics of all the moduli spaces $\mathbf{M}_{g}^{m}$ again in terms of Newton power sum polynomials. It is an extensive generalization of the results of Getzler cited above as well as the formula of Harer and Zagier [34] for the integral Euler characteristics of the moduli space of curves. By using Gorsky's formula, we obtain explicit closed formulas for the equivariant Euler characteristic $e^{\mathfrak{S}_{m}}\left(\mathbf{M}_{g}^{m}\right)$ as well as the generating functions

$$
\sum_{m=0}^{\infty} e\left(\mathbf{M}_{g}^{m} / \mathfrak{S}_{m}\right) x^{m}
$$


for $g \leq 125$. Here we describe the values for $g=3,4 ; m \leq 10$.

$$
\begin{aligned}
& e\left(\mathbf{M}_{3}^{m} / \mathfrak{S}_{m}\right)=3,6,5,2,0,0,1,0,-1,2,2 \quad(m=0,1, \ldots, 10) \\
& e\left(\mathbf{M}_{4}^{m} / \mathfrak{S}_{m}\right)=2,2,0,2,0,2,10,6,-19,-12,34 \quad(m=0,1, \ldots, 10) .
\end{aligned}
$$

In particular $e\left(\mathbf{M}_{3}^{4} / \mathfrak{S}_{4}\right)=e\left(\mathbf{M}_{4}^{2} / \mathfrak{S}_{2}\right)=0$. Thus we find that our results are completely consistent with known results in algebraic geometry, or can be deduced from them by explicit computations. In our forthcoming paper [56], we will further discuss these formulas.

We mention that the integral Euler characteristic of the moduli space $\mathbf{M}_{g}^{m}$, rather than its quotient $\mathbf{M}_{g}^{m} / \mathfrak{S}_{m}$, is known by Harer and Zagier [34] as well as Bini and Harer [2] up to certain values of $g, m$. In particular, $e\left(\mathbf{M}_{3}^{4}\right)=4, e\left(\mathbf{M}_{4}^{2}\right)=-2$.

In our paper [54], we proved that the stable abelianization of $\mathfrak{a}_{g}$ is trivial, namely $H_{1}\left(\mathfrak{a}_{\infty}^{+}\right)^{\mathrm{Sp}}=0$ and deduced from it the vanishing result, $H^{4 g-5}\left(\mathcal{M}_{g} ; \mathbb{Q}\right)=0$, of the top rational cohomology group of the mapping class group $\mathcal{M}_{g}$ of a closed oriented surface of genus $g$ for all $g \geq 2$. See also Church, Farb and Putman [7].

Problem 7.4. For each $k \geq 2$, determine whether the Sp-invariant stable homology group $H_{k}\left(\mathfrak{a}_{\infty}^{+}\right)^{\mathrm{Sp}}$ is finite dimensional or not, especially for the low values $k=2,3, \ldots$.

Remark 7.5. Kontsevich made a conjecture in [38] that the stable homology of each of the infinite dimensional Lie algebras he considered is finite dimensional in each degree. We would like to mention the following implication of this conjecture in the case of $\mathfrak{a}_{g}$. Namely, if $H_{k}\left(\mathfrak{a}_{\infty}^{+}\right)^{\mathrm{Sp}}$ is finite dimensional, then

$$
H_{4 g-4-k}\left(\mathbf{M}_{g} ; \mathbb{Q}\right)=0 \text { for all } g \text { but finitely many possible exceptions. }
$$

This is because, if the assumption is valid, then $P H_{c}^{k}$ is also finite dimensional so that $H_{4 g-4+2 m-k}\left(\mathbf{M}_{g}^{m} ; \mathbb{Q}\right)^{\mathfrak{S}_{m}}=0$ for all $g$ and $m>0$ except for finitely many values. If we put $m=1$ here, we see that $H_{4 g-2-k}\left(\mathbf{M}_{g}^{1} ; \mathbb{Q}\right)=0$ for all $g$ but finitely many exceptions. Now if $H_{4 g-4-k}\left(\mathbf{M}_{g} ; \mathbb{Q}\right) \neq 0$ for some $g \geq 2$, then as was proved in [46] that the homomorphism

$$
H^{*}\left(\mathbf{M}_{g} ; \mathbb{Q}\right) \stackrel{p^{*}}{\rightarrow} H^{*}\left(\mathbf{M}_{g}^{1} ; \mathbb{Q}\right) \stackrel{\cup e}{\longrightarrow} H^{*+2}\left(\mathbf{M}_{g}^{1} ; \mathbb{Q}\right)
$$

is injective for any $g \geq 2$ where $e \in H^{2}\left(\mathbf{M}_{g}^{1} ; \mathbb{Q}\right)$ denotes the Euler class. It follows that $H_{4 g-2-k}\left(\mathbf{M}_{g}^{1} ; \mathbb{Q}\right) \neq 0$ for such $g$. The claim follows.

Looijenga [44] determined the rational homology groups of both of $\mathbf{M}_{3}, \mathbf{M}_{3}^{1}$ and in particular he found an unstable cohomology class in $H^{6}\left(\mathbf{M}_{3} ; \mathbb{Q}\right)$ which is the first known unstable class of the moduli spaces without punctures. He also showed that $H^{8}\left(\mathbf{M}_{3}^{1} ; \mathbb{Q}\right) \cong \mathbb{Q}, H^{6}\left(\mathbf{M}_{3}^{1} ; \mathbb{Q}\right) \cong \mathbb{Q}$. It follows that $P H_{2}\left(\mathfrak{a}_{\infty}^{+}\right)_{10}^{\mathrm{Sp}} \cong \mathbb{Q}$ and $P H_{4}\left(\mathfrak{a}_{\infty}^{+}\right)_{10}^{\mathrm{Sp}} \supset \mathbb{Q}$. It is a very important problem to determine whether $H_{2}\left(\mathfrak{a}_{\infty}^{+}\right)^{\mathrm{Sp}}$ is finite dimensional or not.

Recently Church, Farb and Putman [8] proposed a new stability conjecture about the unstable cohomology of $\operatorname{SL}(n, \mathbb{Z})$, Aut $F_{n}$ and the mapping class groups. In the case of the mapping class groups, our argument above implies the following. Namely, if the above conjecture of Kontsevich is true, then their conjecture also holds in the form that all the groups are trivial. 


\section{DIMENSIONS OF THE Sp-INVARIANT SUBSPACES $\mathfrak{h}_{g, 1}(2 k)^{\mathrm{Sp}}$}

As an application of our consideration, we obtain a complete description of how the Sp-invariant subspaces $\mathfrak{h}_{g, 1}(k)^{\mathrm{Sp}}$ degenerate with respect to $g$. It turns out that this degeneration is perfectly compatible with the orthogonal direct sum decomposition of $\mathfrak{h}_{g, 1}(k)^{\mathrm{Sp}}$ with respect to the canonical metric on it introduced in [53]. The results for all $k \leq 20$ are depicted in Tables 8 and 9 . In the tables, the symbol $*$ denotes that the dimension of the Sp-invariant subspaces stabilizes there with respect to the genus $g$. In general, we can show that the stable range is given by

$$
\begin{aligned}
\operatorname{dim} \mathfrak{h}_{k, 1}(2 k)^{\mathrm{Sp}}=\operatorname{dim} \mathfrak{h}_{k+1,1}(2 k)^{\mathrm{Sp}}=\cdots & (k \text { odd }) \\
\operatorname{dim} \mathfrak{h}_{k-1,1}(2 k)^{\mathrm{Sp}}=\operatorname{dim} \mathfrak{h}_{k, 1}(2 k)^{\mathrm{Sp}}=\cdots & (k \text { even })
\end{aligned}
$$

TABLE 8. Dimensions of $\mathfrak{h}_{g, 1}(2 k)^{\mathrm{Sp}}(\mathrm{i})$

\begin{tabular}{|c|r|r|r|r|r|}
\hline & $g=1$ & $g=2$ & $g=3$ & $g=4$ & $g=5$ \\
\hline $\mathfrak{h}_{g, 1}(2)^{\mathrm{Sp}}$ & $1 *$ & 1 & 1 & 1 & 1 \\
\hline $\mathfrak{h}_{g, 1}(4)^{\mathrm{Sp}}$ & $0 *$ & 0 & 0 & 0 & 0 \\
\hline $\mathfrak{h}_{g, 1}(6)^{\mathrm{Sp}}$ & 1 & 4 & $5 *$ & 5 & 5 \\
\hline $\mathfrak{h}_{g, 1}(8)^{\mathrm{Sp}}$ & 0 & 2 & $3 *$ & 3 & 3 \\
\hline $\mathfrak{h}_{g, 1}(10)^{\mathrm{Sp}}$ & 3 & 51 & 97 & 107 & $108 *$ \\
\hline $\mathfrak{h}_{g, 1}(12)^{\mathrm{Sp}}$ & 0 & 190 & 544 & 643 & $650 *$ \\
\hline $\mathfrak{h}_{g, 1}(14)^{\mathrm{Sp}}$ & 11 & 1691 & 6471 & 8505 & 8795 \\
\hline $\mathfrak{h}_{g, 1}(16)^{\mathrm{Sp}}$ & 10 & 11842 & 69544 & 104190 & 110610 \\
\hline $\mathfrak{h}_{g, 1}(18)^{\mathrm{Sp}}$ & 57 & 100908 & 888099 & 1548984 & 1710798 \\
\hline $\mathfrak{h}_{g, 1}(20)^{\mathrm{Sp}}$ & 108 & 869798 & 12057806 & 25062360 & 29129790 \\
\hline
\end{tabular}

TABLE 9. Dimensions of $\mathfrak{h}_{g, 1}(2 k)^{\mathrm{Sp}}($ ii)

\begin{tabular}{|l|r|r|r|r|}
\hline & $g=6$ & $g=7$ & $g=8$ & $g=9$ \\
\hline $\mathfrak{h}_{g, 1}(14)^{\mathrm{Sp}}$ & 8816 & $8817 *$ & 8817 & 8817 \\
\hline $\mathfrak{h}_{g, 1}(16)^{\mathrm{Sp}}$ & 111131 & $111148 *$ & 111148 & 111148 \\
\hline $\mathfrak{h}_{g, 1}(18)^{\mathrm{Sp}}$ & 1728591 & 1729620 & 1729656 & $1729657 *$ \\
\hline $\mathfrak{h}_{g, 1}(20)^{\mathrm{Sp}}$ & 29688027 & 29728348 & 29729957 & $29729988 *$ \\
\hline
\end{tabular}

Details will be given in our forthcoming paper [57] where we will discuss how the Lie bracket operation on $\mathfrak{h}_{g, 1}^{\mathrm{Sp}}$ is related to the above description of the orthogonal direct sum decomposition as well as the degeneration. This should be important in the investigation of the arithmetic mapping class group.

\section{CONCLUDING REMARKS AND PROBLEMS}

In this section, we discuss differences between the three cases $\mathfrak{g}_{\infty}=\mathfrak{c}_{\infty}, \mathfrak{h}_{\infty, 1}, \mathfrak{a}_{\infty}$. 
Remark 9.1. First we consider the lowest weight part of the continuous cohomology by which we mean the image of the homomorphism

$$
H_{c}^{*}\left(\widehat{\mathfrak{g}}_{\infty}(1)\right)^{\mathrm{Sp}} \rightarrow H_{c}^{*}\left(\mathfrak{g}_{\infty}^{+}\right)^{\mathrm{Sp}}
$$

which is induced by the Lie algebra homomorphism $\mathfrak{g}_{\infty} \rightarrow \mathfrak{g}_{\infty}(1)$. In the commutative case, this lowest weight cohomology is precisely the dual of $\mathcal{A}(\emptyset) \subset H_{*}\left(\mathfrak{c}_{\infty}^{+}\right)^{\mathrm{Sp}}$ so that it is still mysterious. On the other hand, in the other two cases, it is completely understood because the lowest weight cohomology is precisely the totality of $H_{0}$ (Out $\left.F_{n} ; \mathbb{Q}\right)(n \geq$ 2) for the Lie case and the totality of $H_{0}\left(\mathbf{M}_{g}^{m} ; \mathbb{Q}\right)(2 g-2+m>0, m \geq 1)$ for the associative case. We mention here that the lowest weight cohomology of the Lie algebras $\mathfrak{h}_{g, *}^{+}$and $\mathfrak{h}_{g}^{+}$surject onto the tautological algebras $\mathcal{R}^{*}\left(\mathbf{M}_{g}^{1}\right)$ and $\mathcal{R}^{*}\left(\mathbf{M}_{g}\right)$, respectively, by the results of [49],[37]. Further, it was conjectured in [51] that these homomorphisms are isomorphisms.

Remark 9.2. In the commutative case, it is easy to see that the homomorphism $\mathfrak{c}_{g}^{+} \rightarrow$ $\mathfrak{c}_{g}(1)=S^{3} H_{\mathbb{Q}}$ is nothing other than the abelianization of the Lie algebra $\mathfrak{c}_{g}^{+}$because it is fairy easy to see that the Poisson bracket $\mathfrak{c}_{g}(k) \otimes \mathfrak{c}_{g}(1) \rightarrow \mathfrak{c}_{g}(k+1)$ is surjective for any $k \geq 1$. Hence the lowest weight cohomology in this case is the same as those classes which are induced from the abelianization, namely the image of the homomorphism

$$
H_{c}^{*}\left(\widehat{H}_{1}\left(\mathfrak{c}_{\infty}^{+}\right)\right)^{\mathrm{Sp}} \rightarrow H_{c}^{*}\left(\widehat{\mathfrak{c}}_{\infty}^{+}\right)^{\mathrm{Sp}}
$$

Theorem 1.3 shows that this homomorphism is not surjective and furthermore we conjecture that the cokernel is infinitely generated (see Conjecture 5.5). In the associative case, in our former paper [54] we have determined the stable abelianization of $\mathfrak{a}_{\infty}$ which turned out to be very small. Also the associative version of the above homomorphism is far from being surjective. In the Lie case, the known abelianization of $\mathfrak{h}_{\infty, 1}^{+}$turns out to be already very large by [48][11] (although the final answer is not yet known) and many cohomology classes have been defined by making use of it. On the other hand, we proved that $e\left(\right.$ Out $\left.F_{10}\right)=-124$ (Theorem 1.2 (ii)) and it seems that this number is too large to be covered by the above construction. Because of this, we propose the following problem.

Problem 9.3. Prove that the natural homomorphism

$$
H_{c}^{*}\left(\widehat{H}_{1}\left(\mathfrak{h}_{\infty, 1}^{+}\right)\right)^{\mathrm{Sp}} \rightarrow H_{c}^{*}\left(\widehat{\mathfrak{h}}_{\infty, 1}^{+}\right)^{\mathrm{Sp}}
$$

is not surjective.

In view of the fact that a single irreducible piece $\mathfrak{h}_{g}(1)=\left[1^{3}\right]_{\text {Sp }}$ gives rise to the whole tautological algebra $\mathcal{R}^{*}\left(\mathbf{M}_{g}\right)$ as already mentioned in the preceding remark, it seems worthwhile to consider the following problem.

Problem 9.4. Let $\operatorname{Tr}(2 k+1): \mathfrak{h}_{g, 1}^{+} \rightarrow S^{2 k+1} H_{\mathbb{Q}}$ be the $(2 k+1)$-st trace map defined in [48] and let

$$
\lim _{g \rightarrow \infty} P H^{2 n}\left(S^{2 k+1} H_{\mathbb{Q}}\right)_{2 n(2 k+1)}^{\mathrm{Sp}} \rightarrow P H_{c}^{2 n}\left(\widehat{\mathfrak{h}}_{\infty, 1}^{+}\right)_{2 n(2 k+1)}^{\mathrm{Sp}} \cong H_{4 n k}\left(\text { Out } F_{2 n k+n+1} ; \mathbb{Q}\right)
$$


be the homomorphism induced from the above single trace homomorphism. It is easy to see that the left hand side is non-trivial for any $n \geq 1$ and $k \geq 1$. Determine whether the classes in the image of this homomorphism are non-trivial or not.

The case $n=1$ corresponds to the original conjecture proposed in [50] expecting the non-triviality of the classes $\mu_{k}$. In view of our result that $e\left(\operatorname{Out} F_{7}\right)=1$, the case $n=2, k=1$ which asks whether the homomorphism $\lim _{g \rightarrow \infty} P H^{4}\left(S^{3} H_{\mathbb{Q}}\right)_{12}^{\mathrm{Sp}} \cong \mathbb{Q}^{2} \rightarrow$ $P H_{c}^{4}\left(\widehat{\mathfrak{h}}_{\infty, 1}^{+}\right)_{12}^{\mathrm{Sp}} \cong H_{8}\left(\right.$ Out $\left.F_{7} ; \mathbb{Q}\right)$ is non-trivial or not, should be an important test case.

Remark 9.5. In this paper, we consider only the Euler characteristics of various chain complexes. However we are planning to study the boundary operators as well. In fact, we already have a proof of the non-triviality $\mu_{2} \neq 0$, which was first proved by Conant and Vogtmann [12], in our context. Also it will be nice if one could construct cycles corresponding to the unstable cohomology classes found by Looijenga and/or Tommasi.

Remark 9.6. We expect that there should be a close relation between the cohomology of Out $F_{n}$ and that of GL $(n, \mathbb{Z})$. For example, Elbaz-Vincent, Gangl and Soule [15] recently calculated the rational cohomology of $\operatorname{GL}(n, \mathbb{Z})$ for $n=5,6,7$ and it will be a very interesting problem to compare these results with the known results about $H^{*}\left(\right.$ Out $\left.F_{6} ; \mathbb{Q}\right)$. Also we have a conjectural geometric meaning of the classes $\mu_{k} \in H_{4 k}\left(\mathrm{Out} F_{2 k+2} ; \mathbb{Q}\right)$. More precisely, we expect that these classes can be interpreted as certain secondary classes associated with the difference between two reasons for the Borel regulator classes $\beta_{k} \in H^{4 k+1}(\mathrm{GL}(N, \mathbb{Z}) ; \mathbb{R})$ (see Borel [4]) to vanish first in $H^{4 k+1}\left(\right.$ Out $F_{N} ; \mathbb{R}$ ) by the vanishing theorem of Igusa [36] (see also Galatius [20]) and secondly vanish in $H^{4 k+1}\left(\mathrm{GL}\left(N_{k}^{*}, \mathbb{Z}\right) ; \mathbb{R}\right)$ for certain unknown critical rank $N_{k}^{*}$ depending on $k$ (we conjecture that $\left.N_{k}^{*}=2 k+2\right)$. We mention that Bismut and Lott [3] proved that $\beta_{k}$ vanishes in $H^{4 k+1}(\mathrm{GL}(2 k+1, \mathbb{Z}) ; \mathbb{R})$ (we thank Christophe Soule for this information) and the above value for $N_{k}^{*}$ is the next one after $2 k+1$. This expectation is consistent with the result of Conant and Vogtmann [13] where they proved that $\mu_{k}$ vanishes after one stabilization.

Remark 9.7. Our ultimate goal is to enhance our study of the cohomology of the Lie algebra $\mathfrak{h}_{g, 1}$ to those of the groups $\mathcal{H}_{g, 1}^{\text {smooth }}, \mathcal{H}_{g, 1}^{\text {top }}$ of homology cobordism classes of homology cylinders over the surfaces, both in the smooth as well as the topological categories and also the group $\mathrm{Aut}_{0} F_{2 g}^{\mathrm{acy}}$ which was introduced by the second named author in [64]. The group $\mathcal{H}_{g, 1}^{\text {smooth }}$ was introduced by Garoufalidis and Levine [21] as an enlargement of the mapping class group $\mathcal{M}_{g, 1}$. For basic facts as well as various problems about this group, we refer to their paper cited above. We would like to clarify the difference between the above three groups by investigating suitable characteristic classes.

\section{REFERENCES}

1. J. Bergström, Cohomology of moduli spaces of curves of genus three via point counts, J. Reine Angew. Math. 622 (2008) 155-187.

2. G. Bini, J. Harer, Euler characteristics of moduli spaces of curves, J. Eur. Math. Soc. 13 (2011) 487-512. 
3. J. Bismut, J. Lott, Torus bundles and the group cohomology of $G L(N, \mathbb{Z})$, J. Differential Geometry 45 (1997) 196-236.

4. A. Borel, Stable real cohomology of arithmetic groups, Ann. Sci. Ecole Norm. Sup. 7 (1974) 235-272.

5. M. Bridson, K. Vogtmann, Automorphism groups of free groups, surface groups and free abelian groups, in: "Problems on Mapping Class Groups and related Topics", edited by Benson Farb, Proc. Sympos. Pure Math. 74 (2006), American Mathematical Society, 301-316.

6. C. Chevalley, S. Eilenberg, Cohomology theory of Lie groups and Lie algebras, Trans. Amer. Math. Soc. 63 (1948) 85-124.

7. T. Church, B. Farb, A. Putman, The rational cohomology of the mapping class group vanishes in its virtual cohomological dimension, Int. Math. Res. Not. 21 (2012) 5025-5030.

8. T. Church, B. Farb, A. Putman, A stability conjecture for the unstable cohomology of $\mathrm{SL}_{n} \mathbb{Z}$, mapping class groups, and $\operatorname{Aut}\left(F_{n}\right)$, Algebraic topology: applications and new directions, Contemp. Math. 620, Amer. Math. Soc. (2014) 55-70.

9. J. Conant, Ornate necklaces and the homology of the genus one mapping class group, Bull. London Math. Soc. 39 (2007) 881-891.

10. J. Conant, F. Gerlits, K. Vogtmann, Cut vertices in commutative graphs, Q. J. Math. 56 (2005) 321-336.

11. J. Conant, M. Kassabov, K. Vogtmann, Hairy graphs and the unstable homology of $\operatorname{Mod}(g, s), \operatorname{Out}\left(F_{n}\right)$ and $\operatorname{Aut}\left(F_{n}\right)$, J. Topology 6 (2013) 119-153.

12. J. Conant, K. Vogtmann, Morita classes in the homology of automorphism groups of free groups, Geom. Topol. 8 (2004) 121-138.

13. J. Conant, K. Vogtmann, Morita classes in the homology of $\operatorname{Aut}\left(F_{n}\right)$ vanish after one stabilization, Groups Geometry and Dynamics 2 (2008) 1471-1499.

14. M. Culler, K. Vogtmann, Moduli of graphs and automorphisms of free groups, Invent. Math. 84 (1986) 91-119.

15. P. Elbaz-Vincent, H. Gangl, C. Soulé, Perfect forms K-theory and the cohomology of modular groups, Adv. Math. 245 (2013) 587-624.

16. N. Enomoto, T. Satoh, New series in the Johnson cokernels of the mapping class groups of surfaces, Algebr. Geom. Topol. 14 (2014) 627-669.

17. B. Farb (editor), "Problems on Mapping Class Groups and related Topics”, Proc. Sympos. Pure Math. 74, American Mathematical Society, 2006.

18. B. Farb, Some problems on mapping class groups and moduli space, in: "Problems on Mapping Class Groups and related Topics", Proc. Sympos. Pure Math. 74 (2006), American Mathematical Society, 11-55.

19. W. Fulton, J. Harris, "Representation Theory", Graduate Texts in Mathematics 129, Springer Verlag, 1991.

20. S. Galatius, Stable homology of automorphism groups of free groups, Ann. Math 173 (2011) 705-768.

21. S. Garoufalidis, J. Levine, Tree-level invariants of three-manifolds, Massey products and the Johnson homomorphism, in: "Graphs and Patterns in Mathematics and Theoretical Physics", Proc. Sympos. Pure Math. 73 (2005) 173-205.

22. S. Garoufalidis, H. Nakamura, Some IHX-type relations on trivalent graphs and symplectic representation theory, Math. Res. Lett. 5 (1998) 391-402.

23. S. Garoufalidis, T. Ohtsuki, On finite type 3-manifold invariants III: manifold weight systems, Topology 37 (1998) 227-244.

24. I.M. Gelfand, D.I. Kalinin, D.B. Fuks, The cohomology of the Lie algebra of Hamiltonian formal vector fields, (Russian), Funkcional Anal. i Pril. 6 (1972), 25-29; Engl. transl. in Funk. Anal. Appl. 6 (1973), 193-196.

25. F. Gerlits, Invariants in chain complexes of graphs, Ph.D. thesis, Cornell University, 2003.

26. E. Getzler, Topological recursion relations in genus 2, in: "Integrable systems and algebraic geometry (Kobe/Kyoto,1997)", World Sci. Publishing, River Edge, NJ, 1998, 73-106.

27. E. Getzler, Resolving mixed Hodge modules on configuration spaces, Duke. Math. J. 96 (1999) 175-203. 
28. E. Getzler, M.M. Kapranov, Modular operads, Compos. Math. 110 (1998) 65-126.

29. E. Gorsky, On the $S_{n}$-equivariant Euler characteristic of $M_{2, n}$, preprint, arXiv:0707.2662[math.AG].

30. E. Gorsky, The equivariant Euler characteristic of moduli space of curves, Adv. Math. 250 (2014) 588-595

31. J. Gray, On the homology of automorphism groups of free groups, Ph.D. thesis, University of Tennessee, 2011.

32. A. Hamilton, Classes on the moduli spaces of Riemann surfaces through a noncommutative BatalinVilkovisky formalism, Adv. Math. 243 (2013) 67-101.

33. A. Hamilton, A. Lazarev, Characteristic classes of $A_{\infty}$ algebras, J. Homotopy Relat. Struct. 3 (2008) 65-111.

34. J. Harer, D. Zagier, The Euler characteristic of the moduli space of curves, Invent. Math. 85 (1986) 457-485.

35. A. Hatcher, K. Vogtmann, Rational homology of Aut $\left(F_{n}\right)$, Math. Res. Lett. 5 (1998) 759-780.

36. K. Igusa, "Higher Franz-Reidemeister Torsion”, Providence, RI: Amer. Math. Soc., 2002.

37. N. Kawazumi, S. Morita, The primary approximation to the cohomology of the moduli space of curves and stable characteristic classes, Math. Res. Lett. 3 (1996) 629-642.

38. M. Kontsevich, Formal (non)commutative symplectic geometry, in: "The Gel'fand Mathematical Seminars, 1990-1992", Birkhäuser, Boston (1993) 173-187.

39. M. Kontsevich, Feynman diagrams and low-dimensional topology, in: "First European Congress of Mathematics, Vol. II (Paris, 1992)", Progr. Math. 120, Birkhäuser, Basel (1994) 97-121.

40. M. Kontsevich, Rozansky-Witten invariants via formal geometry, Compos. Math. 115 (1999), 115-127.

41. D. Kotschick, S. Morita, The Gelfand-Kalinin-Fuks class and characteristic classes of transversely symplectic foliations, preprint, arXiv:0910.3414[math.SG].

42. T. Le, An invariant of integral homology 3-spheres which is universal for all finite type invariants, Solitons, geometry, and topology: on the crossroad, Amer. Math. Soc. Transl. Ser. 2, 179, Amer. Math. Soc., Providence, RI (1997), 75-100.

43. T. Le, J. Murakami, T. Ohtsuki, On a universal perturbative quantum invariant of 3-manifolds, Topology 37 (1998) 539-574.

44. E. Looijenga, Cohomology of $\mathcal{M}_{3}$ and $\mathcal{M}_{3}^{1}$, in:"Mapping class groups and moduli spaces of Riemann surfaces (Göttingen, 1991/Seattle. WA, 1991)", Cotemp. Math. 150, Amer. Math. Soc., Providence, RI (1993) 205-228.

45. S. Metoki, Non-trivial cohomology classes of Lie algebras of volume preserving formal vector fields, Ph.D. thesis, University of Tokyo, 2000.

46. S. Morita, Characteristic classes of surface bundles, Invent. Math. 90 (1987) 551-577.

47. S. Morita, On the structure and the homology of the Torelli group, Proc. Japan Acad. 65 (1989) 147-150.

48. S. Morita, Abelian quotients of subgroups of the mapping class group of surfaces, Duke Math. J. 70 (1993) 699-726.

49. S. Morita, A linear representation of the mapping class group of orientable surfaces and characteristic classes of surface bundles, Topology and Teichmüller Spaces, World Scientific, 1996, 159-186.

50. S. Morita, Structure of the mapping class groups of surfaces: a survey and a prospect, Geometry and Topology Monographs 2, Proceedings of the Kirbyfest (1999), 349-406.

51. S. Morita, Cohomological structure of the mapping class group and beyond, in: "Problems on Mapping Class Groups and related Topics", edited by Benson Farb, Proc. Sympos. Pure Math. 74 (2006), American Mathematical Society, 329-354.

52. S. Morita, Lie algebra of symplectic derivations and cycles on the moduli spaces, Geometry and Topology Monographs 13 (2008), 335-354.

53. S. Morita, Canonical metric on the space of symplectic invariant tensors and its applications, preprint, arXiv:1404.3354[math.GT].

54. S. Morita, T. Sakasai, M. Suzuki, Abelianizations of derivation Lie algebras of the free associative algebra and the free Lie algebra, Duke Math. J. 162 (2013) 965-1002.

55. S. Morita, T. Sakasai, M. Suzuki, Symmetry of symplectic derivation Lie algebras of free Lie algebras, preprint, arXiv:1109.3963 math.AT]. 
56. S. Morita, T. Sakasai, M. Suzuki, Computation of Gorsky's formula for the equivariant Euler characteristics of the moduli space of curves and its application in the associative graph homology, in preparation.

57. S. Morita, T. Sakasai, M. Suzuki, Structure of symplectic invariant Lie subalgebras of symplectic derivation Lie algebras, preprint, arXiv:1404.3351 [math.AT].

58. R. Ohashi, The rational homology group of $\operatorname{Out}\left(F_{n}\right)$ for $n \leq 6$, Experiment. Math. 17 (2008), 167-179.

59. T. Ohtsuki, Finite type invariants of integral homology 3-spheres, J. Knot. Theory Ramifications 5 (1996), 101-115.

60. T. Ohtsuki (editor), Problems on invariants of knots and 3-manifolds, Geometry and Topology monographs 4, 2003.

61. R.C. Penner, Perturbative series and the moduli space of Riemann surfaces, J. Differential Geometry 27 (1988), 35-53.

62. J. Perchik, Cohomology of Hamiltonian and related formal vector field Lie algebras, Topology 15 (1976), 395-404.

63. R.C. Read, The enumeration of locally restricted graphs (I), J. London Math. Soc. 34 (1959), 417-436.

64. T. Sakasai, Homology cylinders and the acyclic closure of a free group, Alg. Geom. Top. 6 (2006), 603-631.

65. J. Smillie, K. Vogtmann, A generating function for the Euler characteristic of $\operatorname{Out}\left(F_{n}\right)$, J. Pure Appl. Algebra 44 (1987), 329-348.

66. O. Tommasi, Geometry of discriminants and cohomology of moduli spaces, Ph.D. thesis, Radbound University Nijmegen, 2005.

67. O. Tommasi, Rational cohomology of the moduli space of genus 4 curves, Compos. Math. 141 (2005), 359-384.

68. O. Tommasi, Rational cohomology of $\mathcal{M}_{3,2}$, Compos. Math. 143 (2007), 986-1002.

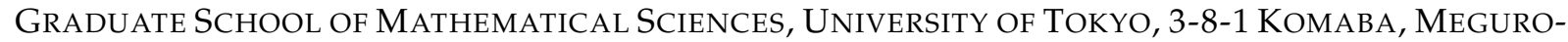
KU, TOKYO, 153-8914, JAPAN

E-mail address: morita@ms.u-tokyo.ac.jp

Graduate School of MATHEMATical Sciences, University of TOKyo, 3-8-1 Komaba, MeguroKU, TOKYO, 153-8914, JAPAN

E-mail address: sakasai@ms.u-tokyo.ac.jp

Department of Frontier Media Science, Meiji University, 4-21-1 Nakano, NaKano-Ku, TOKYO, 164-8525, JAPAN

E-mail address: macky@fms.meiji.ac.jp 